\title{
Light and Laser-based Treatments for Hidradenitis Suppurativa: A Systematic Review
}

\author{
Ilya M. Mukovozov BHSc, MSc, MD, $\mathrm{PhD}^{1^{*}}$, Sara Mirali BSc, PhD ${ }^{2}$, Sophie Khaslavsky BScN ${ }^{3}$, \\ Sunil Kalia MD, MHSc, FAAD, FRCPC ${ }^{1,4,5}$ \\ *Equal contribution \\ ${ }^{1}$ Department of Dermatology and Skin Science, University of British Columbia, Vancouver, BC, Canada \\ ${ }^{2}$ Faculty of Medicine, University of Toronto, Toronto, ON, Canada \\ ${ }^{3}$ Vancouver General Hospital, Vancouver, BC, Canada \\ ${ }^{4}$ Photomedicine Institute and Centre for Clinical Epidemiology \& Evaluation, Vancouver Coastal Health Research \\ Institute, Vancouver, BC, Canada \\ ${ }^{5}$ British Columbia Children's Hospital Research Institute, Vancouver, BC, Canada
}

\section{ABSTRACT}

Background: Hidradenitis suppurativa (HS) is characterized by painful, recurrent lesions occurring mainly in intertriginous areas. The pain, odor, and disfigurement caused by HS significantly impacts quality of life and is challenging to treat. A comprehensive systematic review evaluating the use of light and laser-based treatments for HS is lacking.

Methods: We performed a systematic review by searching Cochrane, MEDLINE and Embase. Title, abstract and full text screening, and data abstraction were done in duplicate.

Results: Forty studies met the inclusion criteria, representing a total of 821 patients. Included studies were comprised of 5 randomized within-patient controlled trials, 1 randomized controlled trial, and 34 case series. Overall, treatments with the most reported cases were laser surgery, photodynamic therapy (PDT), and laser field treatments which showed a response in $80 \%(n=344 / 431), 73 \%(n=122 / 167)$ and $71 \%(n=84 / 101)$ of treated patients respectively. The pooled response rate for psoralen plus ultraviolet A was $69 \%(n=9 / 13)$.

Conclusion: Our results suggest that laser surgery using carbon dioxide $\left(\mathrm{CO}_{2}\right)$ laser or a combination of $\mathrm{CO}_{2}$ and $\mathrm{Nd}$ :YAG lasers has a moderate response rate for HS with the most reported cases. Laser for field treatment and PDT also had moderate response rates with a large number of reported cases. However, extrapolation of these results may be limited due to the majority of the studies being case series, lack of standardized outcomes being assessed, and insufficient long term follow up results.

\section{INTRODUCTION}

Hidradenitis suppurativa (HS) is characterized by painful, recurrent papules and nodules occurring mainly in intertriginous areas. The pain, odor, and disfigurement caused by HS significantly impacts patients' quality of life and is associated with increased rates of anxiety and depression. ${ }^{1-3}$ Moreover, the symptoms of HS can be physically limiting and interfere with employment and personal functioning. ${ }^{4}, \quad 5$ Early diagnosis and management of HS reduces the risk of disease progression. ${ }^{6}$ Treatment for HS are often multimodal and include medical, surgical, and laser and light-based therapies. Although treatment with tumor necrosis factor inhibitors, interleukin-1 inhibitors, antibiotics and others have been reported, these 
treatments may be associated with significant cost, adverse effects, declining efficacy, and possible drug-drug interactions. In practice, patients may express the preference to avoid systemic treatments if possible. Light and laser-based management is known to be a safe and effective treatment option for many dermatological conditions. Furthermore, light and laser-based treatment provides the advantage of avoiding the adverse effects and drug interactions associated with systemic therapies. Light and laser-based treatments commonly employed for HS include psoralen plus ultraviolet A (PUVA), photodynamic therapy (PDT), neodymiumdoped yttrium aluminum garnet (Nd:YAG) laser, carbon dioxide $\left(\mathrm{CO}_{2}\right)$ laser, and others $^{7-10}$. These treatments are thought to reduce HS lesions by debulking tissue and by reducing the amount of hair follicles, sebaceous glands, and bacterial load. ${ }^{11}$ Although laser and light-based treatments for HS have been reported, little is known about their comparative effectiveness. The aim of this systematic review is to summarize outcomes for light and laser-based treatments used for HS, enabling physicians to better predict clinical response.

\section{METHODS}

A systematic review of the literature was conducted adhering to PRISMA reporting guidelines. ${ }^{12}$ The study protocol was registered in the PROSPERO database (CRD42020223612).

\section{Study eligibility criteria}

Eligibility criteria for this review were:

- Population: individuals of any age and sex with HS

- Intervention (exposure): Nd:YAG laser, PDT, $\mathrm{CO}_{2}$ laser, intense pulse light (IPL), PUVA
- Comparator: patients with HS not exposed to intervention

- Outcomes: pain, lesion count, hidradenitis severity score (HSS), visual analogue scale (VAS), dermatology life quality index (DLQI), recurrence, healing time, physician global assessment (PGA), sartorius score, lesion area and severity index (LASI), patient satisfaction, clinical response

- Study design: cohort, cross-sectional, and case-series

\section{Literature search and screening}

MEDLINE, Embase, the Cochrane Database of Systematic Reviews, and PubMed were searched on June 12, 2020 using variations of the keywords "hidradenitis suppurativa" and "light" (Tables S1-S4). No date or language restrictions were applied. Title, abstract, and full-text screening were conducted by two independent reviewers (I.M., S.M.) using Covidence online systematic review software (www.covidence.org). Any conflicts between reviewers were resolved by discussion until a consensus was reached.

\section{Data extraction}

Data extraction was completed by three independent reviewers (I.M., S.M., S.K.) on a standardized extraction form.

\section{Level of evidence assessment}

Level of evidence was assessed using a modified hierarchy proposed by Guyatt and Sackett, where: (1) prospective controlled trial; (2) retrospective study or large case series; (3) small case series. ${ }^{13}$

\section{Data synthesis}


After data collection, we determined that quantitative evidence synthesis was not feasible due to differences between the studies included in our review. Specifically, differences in study design, HS severity, heterogeneity in treatment modalities, patient populations, and outcome measurement. Instead, our results are presented in narrative form for each outcome

\section{RESULTS}

\section{Study selection}

Our literature search yielded 460 articles, 405 of which were excluded based on title and abstract review (Figure 1). Of the 55 studies retrieved for full text screening, 15 were excluded. A total of 40 studies were ultimately included in the review, 6 of which were conference abstracts.

The included studies were published between 1987 and 2020 and included 5 randomized within-patient controlled trials, 1 randomized controlled trial, and 34 case series (Table 1). The majority of studies were conducted in Spain $(n=7)$, North America $(n=6)$, United Kingdom $(n=5)$, and Italy $(n=4)$ (Table 1).

\section{Patient characteristics (age/sex)}

In total, our pooled analysis includes 821 patients, $87 \% \quad(n=623 / 716)$ females, with mean age of 33 years, ranging from 15 to 73 years of age.

\section{Targeted phototherapy}

A total of 22 studies ${ }^{14-35}$ included in our review reported on targeted phototherapy using lasers and energy-based devices in patients with HS, representing a sample size of 724 patients with a mean age of 37.2 years (range: $14-73)$, and $76.3 \% \quad\left(n=497 / 651^{\dagger}\right)$ were female. Overall, targeted phototherapy improved lesions in $77.6 \%(n=413 / 532)$ of HS patients. Adverse effects were reported in $22.9 \%(n=122 / 532)$ of treated patients (Table 2).

$\mathrm{CO}_{2}$

$\mathrm{CO}_{2}$ laser was employed in 10 studies $(n=396)$, with $78.5 \%(n=311 / 396)$ of patients showing improvement (Table 2). ${ }^{15-17,}$ 19-21, 2426, 28 Adverse events were reported in $26.2 \%$ of cases $(n=103 / 393)$. All 10 studies found that $\mathrm{CO}_{2}$ laser was an effective treatment modality. In a study of 24 patients, Lapins et al. showed that $\mathrm{CO}_{2}$ laser treatment with healing by secondary intention was safe, beneficial, and rapidly effective with a mean resolution period of 4 weeks. The majority of patients $(91.7 \%, n=22 / 24)$ experienced no recurrence in treated areas. Overall, all patients reported satisfaction with this treatment modality. ${ }^{24}$ The treatments were well tolerated in all patients, and average healing time post procedure was 8.8 weeks. ${ }^{21}$ Among patients with recurrent HS, Mikkelsen et al. found that $\mathrm{CO}_{2}$ laser surgery with healing by secondary intention was effective with high patient satisfaction rates $(94.8 \%$, $\mathrm{n}=55 / 58)$. Improvement was reported as great in $75.8 \%(n=44 / 58)$ of patients and mild in $19.0 \% \quad(n=11 / 58)$. Overall, $91.4 \%$ $(n=53 / 58)$ of patients stated they would recommend the procedure. ${ }^{28}$

\section{Nd:YAG}

Four studies consisting of 92 patients reported on the use of $\mathrm{Nd}: Y A G$ in $\mathrm{HS}^{27,29,32 \text {, }}$ 33. Among studies with reported outcomes, $85 \%(n=30 / 35)$ of treated patients showed improvement (Table 2) and adverse events were reported in $15 \%$ of cases $(n=8 / 53)$. Overall, 3 of the 4 studies found that Nd:YAG laser 


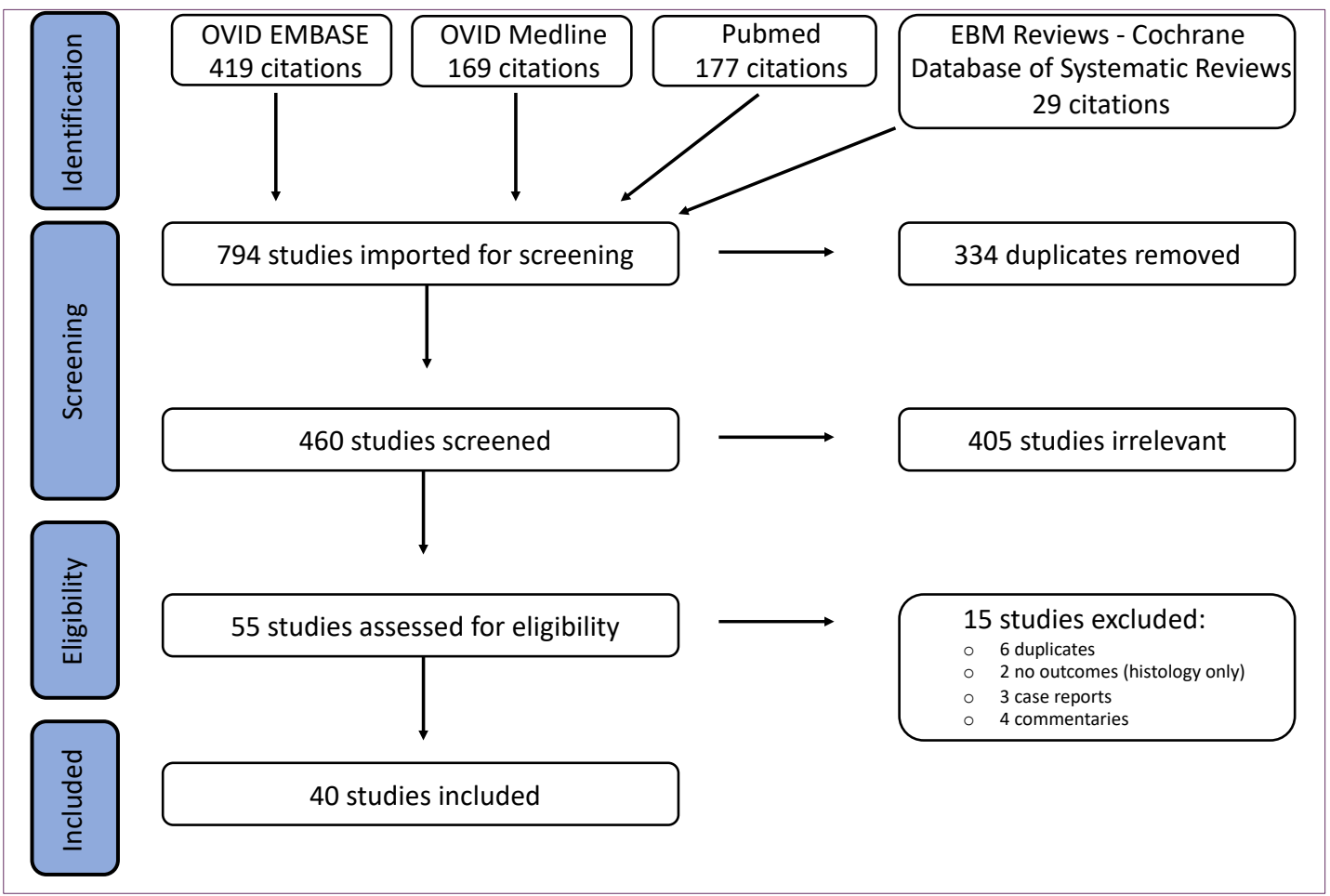

Figure 1: PRISMA diagram of study selection process

treatment improved HS. ${ }^{27,} 32,33$ In a study of 15 HS patients, Vossen et al. found that Nd:YAG for hair removal prevented disease progression. Patients reported a decrease in the number of monthly flares and average HS disease severity, as measured by a numerical response scale (NRS), was significantly lower post treatment (NRS $6.4 \pm$ 2.8 vs NRS $3.6 \pm 3.5)$. Overall, $67 \%$ $(n=10 / 15)$ of patients recommended the treatment. $^{32}$

\section{$\mathrm{Nd}: \mathrm{YAG} / \mathrm{CO}_{2}$ combined}

Two studies combined Nd:YAG and $\mathrm{CO}_{2}$ laser treatment $(n=58)$, with both reporting a benefit in HS (Table 3). ${ }^{14}, 23$ In a study comparing $\mathrm{Nd}$ :YAG laser with combined $\mathrm{CO}_{2}$ and Nd:YAG lasers in 20 patients, combination treatment resulted in better outcomes compared to Nd:YAG alone (mean improvement in $\mathrm{CO}_{2}$ and $\mathrm{Nd}: \mathrm{YAG}: 90 \% \pm$ 20.52 vs mean improvement in Nd:YAG:
$70.68 \% \quad \pm$

23.55).

\section{Other lasers}

Two studies $(n=31)$ reported on the use of 'other' lasers, including PEHT (Pediatric Endoscopic Hidradenitis Treatment) and diode laser in HS patients. ${ }^{18,34}$ Collectively, treatment showed improvement in 93.5\% $(n=29 / 31)$ of patients studied. In a study by Esposito et al., PEHT was found to be an effective treatment for $\mathrm{HS}$ and patients reported satisfaction with results. Recurrence in untreated locations was reported in only $18 \% \quad(n=2 / 11)$ of patients, which were successfully resolved using the same treatment. ${ }^{34}$ In a study by Fabbrocini et al., diode laser reduced Sartorius score, improved HiSCR, and reduced DLQI. Overall, $90 \%$ of patients experienced improvement, with $5 \% \quad(n=1 / 20)$ having complete response, 35\% $(n=7 / 20)$ good response, $50 \%(n=10 / 20)$ partial response,

November $2021 \quad$ Volume 5 Issue 6 
and $10 \% \quad(n=2 / 20)$ no response. The Sartorius score improved from $28.6 \pm 13.0$ at baseline to $19.8 \pm 12.3$ post-treatment. ${ }^{18}$

IPL

Four studies $(n=83)$ in our review assessed the use of IPL for the treatment of $\mathrm{HS}$. Overall, IPL led to improvement in 65\% $(n=54 / 83)$ of patients and adverse events were reported in $25 \%(n=16 / 64)$ (Table 2). ${ }^{22}$, $30,31,35$ Highton et al., reported that IPL treatment resulted in long-term clinical improvement of lesions in $17 \mathrm{HS}$ patients in an intra-individual controlled trial. Clinical improvement was also corroborated by independent analysis of clinical photographs and patient reported satisfaction. ${ }^{22}$ Another study of $25 \mathrm{HS}$ patients found that IPL was useful as an adjuvant treatment for HS patients with mild to moderate disease, and $52 \%(n=13 / 25)$ of patients reported reduced disease activity. ${ }^{31}$

\section{Photodynamic therapy (PDT)}

17 studies $^{8,36-51}$ reported on the use of PDT in patients with HS $(n=167)$. The mean age was 33.3 years (range: 17-62) and 68.9\% $(n=115 / 167)$ were female. Overall, PDT was effective in $70.7 \%(n=118 / 167)$ of patients. Adverse events were screened for in $38.3 \%$ $(n=64 / 167)$ of the PDT cohort, and were reported in 35.9\% $(n=23 / 64)$.

A number of photosensitizers were used in PDT. Ten studies $(n=121)^{8}, 37,43-46,48-51$ reported on PDT for HS with the photosensitizer aminolevulinic acid (ALA). In this cohort, $78.5 \% \quad(n=95 / 121)$ of treated patients responded. Suarez-Valladares et al. found that in a series of HS patients treated with intralesional ALA-PDT, 76.3\% $(n=29 / 38)$ achieved complete remission. ${ }^{8}$ In a smaller study of five Hurley stage II or III HS patients with recalcitrant disease, no significant improvement was seen, despite mild improvements in Sartorius score, VAS, and DLQI in all patients (mean change from baseline to post-treatment: Sartorius $=-1.6$, VAS $=-0.3$, DLQI $=-6.4 \%)$. Swelling and blistering was present in $40 \%(n=2 / 5)$ of patients after the first treatment for 8-10 days. 52

Other photosensitizers utilized for PDT in HS include methylene blue, methyl amino levulinate, and tetracycline. Overall, four studies consisting of 28 patients were included in this cohort. ${ }^{36,38,41,42}$ Of those that reported individual patient outcomes, 94.4\% ( $n=17 / 18)$ of patients reported improvement. Adverse effects were screened in $72.2 \%$ $(n=13 / 18)$ of patients and of these, adverse effects were reported in $92.3 \% \quad(n=12 / 13)$. Agut-Busquet et al. followed 7 HS Hurley stage II and III patients treated with PDT and methylene blue. PDT and methylene blue treatment improved DLQI and reduced lesion size in $85.7 \%(n=6 / 7)$ of patients after 1 month. ${ }^{36}$ Relapse was reported in $28.6 \%$ $(n=2 / 7)$ at 7 and 12 weeks post-treatment.

\section{Psoralen plus ultraviolet A (PUVA)}

PUVA to treat HS was examined in a single retrospective chart review. ${ }^{7}$ A total of 13 patients were treated with a regimen involving a 15 min bath containing $30 \mathrm{~mL}$ of 1-2\% 8-methoxypsoralen lotion followed by broadband ultraviolet $A$. Treatments were performed twice weekly, with a median of 25 (range: 3-57) treatments. Overall, five patients were reported to have "clear or near clear' HS, four patients reached 'moderate clearance', and four showed 'no to minimal' improvement. Overall, bath PUVA was effective in $69 \%(n=9 / 13)$ of patients in this series and was well tolerated. Adverse events were recorded for two patients and included erythema and claustrophobia. 


\section{DISCUSSION}

The studies included in our review reported on patients with both moderate and advanced disease. Many of the studies included in our review utilize laser-based treatments for surgery of affected sites in patients with advanced disease resistant to other therapies. Our results suggest that laser-based surgery is associated with a moderate response rate based on a large sample of pooled patients. Furthermore, many of the studies employing laser devices for surgery of HS lesions included patients with long standing and recalcitrant disease, suggesting that these treatment modalities can be employed an adjuncts with success even in patients with advanced disease.

Likewise, treatment modalities that utilize laser-based field therapy of affected areas using Nd:YAG, IPL, or diode laser were associated with a mild response rate based on a large sample of pooled patients. Laser field treatments for HS were employed in patients with both early and advanced disease, suggesting that this treatment modality is appropriate both to treat advanced disease and as a prophylactic therapy to target follicular inflammation and prevent disease progression.

A number of adverse events were reported in the studies included in our review. Overall, PDT was associated with the highest rates of reported adverse events (36\%). Laser field treatments had less reported adverse events compared to lasers employed for surgical treatment of HS lesions (18\% vs $25 \%$ ). A number of factors may contribute to this observed difference, including differences in study quality and reporting of adverse events, given that only 105 out of 424 patients treated with laser surgery for HS had adverse events recorded. Some of the adverse events reported for laser surgery included pain, infection, hypertrophic scaring, contracture and wound dehiscence. Side effects associated with PDT included pain, erythema, blistering, burning. Bath PUVA was generally well tolerated, although two patients reported erythema and claustrophobia. Collectively, these findings suggest that while light therapy is a moderately effective treatment modality, adverse events are not uncommon, and patients should be counselled on the risks and benefits of light therapy.

While the mechanism underlying the effectiveness of light-based treatments for HS are not well understood, several possibilities have been suggested. $\mathrm{CO}_{2}$ laser vaporization and excision removes epithelial sinus tracts which may contain debris and bacteria that can lead to relapse. ${ }^{53}$ Nonablative lasers cause thermal damage to the dermis, which may initiate a wound-healing response with upregulation of procollagen mRNA, matrix metalloproteinases, and cytokines that contribute to wound healing. ${ }^{54}$

Our study has several limitations. Many studies did not report on the side effects of light-based therapies, which restricts our analysis on treatment tolerability. Secondly, some studies reported aggregate data and outcomes for individual patients could not be extracted. Moreover, different outcome measures were used in the studies included in our review, further challenging comparisons between studies. Finally, the majority of the included studies were case series, which limits the generalizability of our analysis.

\section{CONCLUSION}

Despite these limitations, our review provides important information that must be 
interpreted in a clinical context. First, lightbased treatments show moderate benefit in both early and advanced disease. Second, laser devices and PDT have moderate clearance rates for patients with $\mathrm{HS}$, however access to the technology and impractical treatment delivery (especially with extensive disease) may be a barrier. Third, adverse events are not uncommon with light therapy and patients should be counselled on these risks. Though randomized control trials would be the ideal way to validate our findings, studies with treatment comparators are needed to further delineate the therapeutic ladder for HS.

\section{Conflict of Interest Disclosures: None}

Funding: None

\section{Corresponding Author:}

Ilya M. Mukovozov, MD, PhD

Skin Care Centre

835 W 10th Ave,

Vancouver, BC V5Z 4E8

Phone: (604) 875-5151

Email: ilya.mukovozov@alumni.ubc.ca

\section{References:}

1. Esmann S, Jemec GB. Psychosocial impact of hidradenitis suppurativa: a qualitative study. Acta Derm Venereol. May 2011;91(3):328-32. doi:10.2340/00015555-1082

2. Matusiak L, Szczech J, Bieniek A, NowickaSuszko D, Szepietowski JC. Increased interleukin (IL)-17 serum levels in patients with hidradenitis suppurativa: Implications for treatment with antiIL-17 agents. Journal of the American Academy of Dermatology. Apr 2017;76(4):670-675. doi:10.1016/j.jaad.2016.10.042

3. Wright S, Strunk A, Garg A. New Onset Depression among Children, Adolescents, and Adults with Hidradenitis Suppurativa. J Am Acad Dermatol. May 2020;doi:10.1016/j.jaad.2020.05.090

4. Alavi A, Anooshirvani N, Kim WB, Coutts P, Sibbald RG. Quality-of-life impairment in patients with hidradenitis suppurativa: a Canadian study. Am J Clin Dermatol. Feb 2015;16(1):61-5. doi:10.1007/s40257-014-0105-5
5. von der Werth JM, Jemec GB. Morbidity in patients with hidradenitis suppurativa. $\mathrm{Br} \mathrm{J}$ Dermatol. Apr 2001;144(4):809-13. doi:10.1046/j.1365-2133.2001.04137.x

6. Saunte DM, Boer J, Stratigos A, et al. Diagnostic delay in hidradenitis suppurativa is a global problem. Br J Dermatol. Dec 2015;173(6):1546-9. doi:10.1111/bjd.14038

7. Shareef M, Dawe R. Bath psoralen plus ultraviolet $A$ for hidradenitis suppurativa: a review of 13 patients. Br J Dermatol. Apr 2011;164(4):895-6. doi:10.1111/j.13652133.2010.10193.x

8. Suárez Valladares MJ, Eiris Salvado N, Rodríguez Prieto MA. Treatment of hidradenitis suppurativa with intralesional photodynamic therapy with 5-aminolevulinic acid and 630nm laser beam. J Dermatol Sci. Mar 2017;85(3):241246. doi:10.1016/j.jdermsci.2016.12.014

9. Grimstad O, Tzellos T, Dufour DN, et al. Evaluation of medical and surgical treatments for hidradenitis suppurativa using real-life data from the Scandinavian registry (HISREG). Journal of the European Academy of Dermatology and Venereology. Jun 2019;33(6):1164-1171. doi:10.1111/jdv.15353

10. Mahmoud BH, Tierney E, Hexsel CL, Pui J, Ozog DM, Hamzavi IH. Prospective controlled clinical and histopathologic study of hidradenitis suppurativa treated with the long-pulsed neodymium:yttrium-aluminium-garnet laser. Journal of the American Academy of Dermatology. Apr 2010;62(4):637-645. doi:10.1016/j.jaad.2009.07.048

11. Hamzavi IH, Griffith JL, Riyaz F, Hessam S, Bechara FG. Laser and light-based treatment options for hidradenitis suppurativa. J Am Acad Dermatol. Nov 2015;73(5 Suppl 1):S78-81. doi:10.1016/j.jaad.2015.07.050

12. Moher D, Liberati A, Tetzlaff J, Altman DG, Group P. Preferred reporting items for systematic reviews and meta-analyses: the PRISMA statement. BMJ. Jul 2009;339:b2535. doi:10.1136/bmj.b2535

13. Guyatt GH, Sackett DL, Sinclair JC, Hayward R, Cook DJ, Cook RJ. Users' guides to the medical literature. IX. A method for grading health care recommendations. Evidence-Based Medicine Working Group. JAMA. Dec 1995;274(22):18004. doi:10.1001/jama.274.22.1800

14. Abdel Azim AA, Salem RT, Abdelghani R. Combined fractional carbon dioxide laser and long-pulsed neodymium : yttrium-aluminiumgarnet $(1064 \mathrm{~nm})$ laser in treatment of hidradenitis suppurativa; a prospective randomized intra-individual controlled study.

November $2021 \quad$ Volume 5 Issue 6 
International journal of dermatology.

2018;57(9):1135-1144.

doi:https://dx.doi.org/10.1111/ijd.14075

15. Braunberger TL, Vakharia $P$, Nicholson CL, Parks-Miller A, Hamzavi IH. CARBON DIOXIDE LASER EXCISION IN HIDRADENITIS SUPPURATIVA: DEMOGRAPHICS, COMORBIDITIES, HEALING TIME AND COMPLICATIONS. Lasers in Surgery and Medicine. Apr 2018;50(4):360-360.

16. Crocco El, Dalapicola MC, Suzuki NM, Alves RO. Surgical Treatment of Chronic Hidradenitis Suppurativa: CO2 Laser Stripping-Second Intention Technique. Dermatologic Surgery. Mar 2016;42(3):429-431. doi:10.1097/dss.0000000000000637

17. Dalrymple JC, Monaghan JM. TREATMENT OF HIDRADENITIS SUPPURATIVA WITH THE CARBON-DIOXIDE LASER. British Journal of Surgery. May 1987;74(5):420-420. doi:10.1002/bjs.1800740535

18. Fabbrocini G, França K, Lotti T, et al. Intralesional Diode Laser $1064 \mathrm{~nm}$ for the Treatment of Hidradenitis Suppurativa: A Report of Twenty Patients. Open Access Maced J Med Sci. Jan 2018;6(1):31-34. doi:10.3889/oamjms.2018.045

19. Finley EM, Ratz JL. Treatment of hidradenitis suppurativa with carbon dioxide laser excision and second-intention healing. Journal of the American Academy of Dermatology. Mar 1996;34(3):465-469. doi:10.1016/s01909622(96)90441-7

20. Grimstad $\varnothing$, Tzellos T, Dufour DN, et al. Evaluation of medical and surgical treatments for hidradenitis suppurativa using real-life data from the Scandinavian registry (HISREG). J Eur Acad Dermatol Venereol. Jun 2019;33(6):1164-1171. doi:10.1111/jdv.15353

21. Hazen PG, Hazen BP. Hidradenitis Suppurativa: Successful Treatment Using Carbon Dioxide Laser Excision and Marsupialization. Dermatologic Surgery. Feb 2010;36(2):208-213. doi:10.1111/j.1524-4725.2009.01427.x

22. Highton L, Chan WY, Khwaja N, Laitung JKG. Treatment of Hidradenitis Suppurativa with Intense Pulsed Light: A Prospective Study. Plastic and Reconstructive Surgery. Aug 2011;128(2):459-465. doi:10.1097/PRS.0b013e31821e6fb5

23. Jain $V$, Jain $A$. Use of lasers for the management of refractory cases of hidradenitis suppurativa and pilonidal sinus. J Cutan Aesthet Surg. Jul 2012;5(3):190-2. doi:10.4103/0974-2077.101377

24. Lapins J, Marcusson JA, Emtestam L. SURGICAL-TREATMENT OF CHRONIC
HIDRADENITIS SUPPURATIVA - CO2-LASER

STRIPPING-SECONDARY INTENTION

TECHNIQUE. British Journal of Dermatology. Oct 1994;131(4):551-556.

25. Lapins J, Sartorius K, Emtestam L. Scannerassisted carbon dioxide laser surgery: a retrospective follow-up study of patients with hidradenitis suppurativa. J Am Acad Dermatol. Aug 2002;47(2):280-5. doi:10.1067/mjd.2002.124601

26. Madan V, Hindle E, Hussain W, August PJ. Outcomes of treatment of nine cases of recalcitrant severe hidradenitis suppurativa with carbon dioxide laser. British Journal of Dermatology. Dec 2008;159(6):1309-1314. doi:10.1111/j.1365-2133.2008.08932.x

27. Mahmoud BH, Tierney E, Hexsel CL, Pui J, Ozog DM, Hamzavi IH. Prospective controlled clinical and histopathologic study of hidradenitis suppurativa treated with the long-pulsed neodymium:yttrium-aluminium-garnet laser. J Am Acad Dermatol. Apr 2010;62(4):637-45. doi:10.1016/j.jaad.2009.07.048

28. Mikkelsen PR, Dufour DN, Zarchi K, Jemec GBE. Recurrence Rate and Patient Satisfaction of $\mathrm{CO} 2$ Laser Evaporation of Lesions in Patients With Hidradenitis Suppurativa: A Retrospective Study. Dermatologic Surgery. Feb 2015;41(2):255-260. doi:10.1097/dss.0000000000000264

29. Naouri M, Maruani A, Lagrange S, et al. Treatment of hidradenitis suppurativa using a long-pulsed hair removal neodymium:yttriumaluminium-garnet laser: A multicenter, prospective, randomized, intraindividual, comparative trial. J Am Acad Dermatol. Jan 2021;84(1):203-205.

doi:10.1016/j.jaad.2020.04.117

30. Piccolo D, Di Marcantonio D, Crisman G, et al. Unconventional use of intense pulsed light. Biomed Res Int. 2014;2014:618206. doi:10.1155/2014/618206

31. Riis PT, Saunte DM, Sigsgaard V, Wilken C, Jemec GBE. Intense pulsed light treatment for patients with hidradenitis suppurativa: beware treatment with resorcinol. Journal of Dermatological Treatment. 2018;29(4):385-387. doi:10.1080/09546634.2017.1387226

32. Vossen A, van der Zee HH, Terian M, van Doorn MBA, Prens EP. Laser hair removal alters the disease course in mild hidradenitis suppurativa. Journal Der Deutschen Dermatologischen Gesellschaft. Jul 2018;16(7):901-903. doi:10.1111/ddg.13563

33. Xu LSY, Wright DR, Mahmoud BH, Ozog DM, Mehregan DA, Hamzavi IH. Histopathologic Study of Hidradenitis Suppurativa Following November 2021 Volume 5 Issue 6 
Long-Pulsed 1064-nm Nd:YAG Laser Treatment. Archives of Dermatology. Jan 2011;147(1):21-28. doi:10.1001/archdermatol.2010.245

34. Esposito C, Del Conte F, Cerulo M, et al. Pediatric Endoscopic Hidradenitis Treatment: A New Minimally Invasive Treatment for Pediatric Patients with Hidradenitis Suppurativa. Journal of Laparoendoscopic \& Advanced Surgical Techniques. Apr 2020;30(4):464-470. doi:10.1089/lap.2019.0614

35. Wilden S, Friis M, Tuettenberg A, et al. Combined treatment of hidradenitis suppurativa with intense pulsed light (IPL) and radiofrequency (RF). Journal of Dermatological Treatment. 2019; ((Wilden, Friis, Tuettenberg, StaubachRenz, Wegner, Grabbe, von Stebut) Department of Dermatology, University Medical Center, Johannes Gutenberg, University, Mainz, Germany(von Stebut) Department of Dermatology, Faculty of Medicine, University of Cologne)doi:http://dx.doi.org/10.1080/09546634. 2019.1677842

36. Agut-Busquet E, Romani J, Gilaberte Y, GarciaMalinis A, Ribera-Pibernat M, Luelmo J. Photodynamic therapy with intralesional methylene blue and a $635 \mathrm{~nm}$ light-emitting diode lamp in hidradenitis suppurativa: a retrospective follow-up study in 7 patients and a review of the literature. Photochemical \& Photobiological Sciences. 2016;15(8):1020-1028. doi:10.1039/c6pp00082g

37. Navarrete RA, Nisis AH, Cares JP. Effectiveness of 5-Aminolevulinic Acid Photodynamic Therapy in the Treatment of Hidradenitis Suppurativa: A Report of 5 Cases. Actas Dermo-Sifiliograficas. Jul-Aug 2014;105(6):614-617. doi:10.1016/j.ad.2013.10.016

38. Belotto RA, Tardivo JP, Baptista MS, Santos RE. CLINICAL RESPONSE OF HIDRADENITIS SUPPURATIVA TO PHOTODYNAMIC THERAPY USING TETRACYCLINE AND RL50 (R) LIGHT SOURCE. Lasers in Surgery and Medicine. Mar 2013;45:62-63.

39. Bu WB, $X u X L$, Wang $Y$, et al. Surgery combined with photodynamic therapy for the treatment of Hidradenitis Suppurativa: A report of 7 cases. Photodiagnosis and Photodynamic Therapy. Jun 2017;18:46-49. doi:10.1016/j.pdpdt.2017.01.176

40. Calzavara-Pinton PG, Rossi MT, Aronson E, Sala $\mathrm{R}$, Italian Grp Photodynamic T. A retrospective analysis of real-life practice of off-label photodynamic therapy using methyl aminolevulinate (MAL-PDT) in 20 Italian dermatology departments. Part 1: Inflammatory and aesthetic indications. Photochemical \&
Photobiological Sciences. 2013;12(1):148-157. doi:10.1039/c2pp25124h

41. Fadel MA, Tawfik AA. New topical photodynamic therapy for treatment of hidradenitis suppurativa using methylene blue niosomal gel: a singleblind, randomized, comparative study. Clinical and Experimental Dermatology. Mar 2015;40(2):116-122. doi:10.1111/ced.12459

42. Abstracts of the 8th European Hidradenitis Suppurativa Foundation (EHSF) Conference, 6-8 February 2019, Wrocław, Poland. Exp Dermatol. Feb 2019;28 Suppl 2:5-55.

doi:10.1111/exd.13893

43. Passeron T, Khemis A, Ortonne JP. Pulsed dye laser-mediated photodynamic therapy for acne inversa is not successful: A pilot study on four cases. Journal of Dermatological Treatment. 2009;20(5):297-298. Pii 910443295. doi:10.1080/09546630902882063

44. Rodriguez-Prieto MA, Valladares-Narganes LM, Gonzalez-Sixto B, Noguerol-Cal M. Efficacy of intralesional photodynamic therapy for the treatment of hidradenitis suppurativa. Journal of the American Academy of Dermatology. May 2013;68(5):873-875. doi:10.1016/j.jaad.2012.11.030

45. Sotiriou E, Apalla Z, Maliamani F, loannides D. Treatment of recalcitrant hidradenitis suppurativa with photodynamic therapy: report of five cases. Clin Exp Dermatol. Oct 2009;34(7):e235-6. doi:10.1111/j.1365-2230.2008.03094.x

46. Strauss RM, Pollock B, Stables GI, Goulden V, Cunliffe WJ. Photodynamic therapy using aminolaevulinic acid does not lead to clinical improvement in hidradenitis suppurativa. British Journal of Dermatology. Apr 2005;152(4):803804. doi:10.1111/j.1365-2133.2005.06475.x

47. Suarez-Valladares $M$, Espasandin-Arias $M$, Varas-Meis E, Fernandez-Canga P, CastineirasGonzalez J, Rodriguez-Prieto M. Effectiveness of intralesional photodynamic therapy in the management of Hidradenitis Suppurativa. Experimental Dermatology. May 2018;27:31-32.

48. Valladares-Narganes LM, Rodriguez-Prieto MA, Blanco-Suarez MD, Rodriguez-Lage C, GarciaDoval I. Treatment of hidradenitis suppurativa with intralesional photodynamic therapy using a laser diode attached to an optical cable: a promising new approach. British Journal of Dermatology. Apr 2015;172(4):1136-1139. doi:10.1111/bjd.13385

49. Vilarrasa E, Bittencourt F, Martorell A, Puig L. Photodynamic therapy in the treatment of moderate-to-severe hidradenitis suppurativa: a report of 28 patients. Experimental Dermatology. Feb 2019;28:49-49.

November $2021 \quad$ Volume 5 Issue 6 
50. Zhang LL, Wang PR, Shi L, et al. Topical 5aminolevulinic acid photodynamic therapy improved refractory acne conglobata and perifolliculitis capitis abscedens et suffodiens rather than hidradenitis suppurativa. Journal of Innovative Optical Health Sciences. Jan 2016;9(1)1640002. doi:10.1142/s1793545816400022

51. Gold M, Bridges TM, Bradshaw VL, Boring M. ALA-PDT and blue light therapy for hidradenitis suppurativa. J Drugs Dermatol. 2004 Jan-Feb 2004;3(1 Suppl):S32-5.

52. Sotiriou E, Apalla Z, Maliamani F, loannides D. Treatment of recalcitrant hidradenitis suppurativa with photodynamic therapy: report of five cases. Clinical and Experimental Dermatology. Oct 2009;34(7):e235-e236. doi:10.1111/j.13652230.2008.03094.x

53. Lapins J, Sartorius K, Emtestam L. Scannerassisted carbon dioxide laser surgery: A retrospective follow-up study of patients with hidradenitis suppurativa. Journal of the American Academy of Dermatology. Aug 2002;47(2):280285. doi:10.1067/mjd.2002.124601

54. Orringer JS, Voorhees JJ, Hamilton T, et al. Dermal matrix remodeling after nonablative laser therapy. J Am Acad Dermatol. Nov 2005;53(5):775-82.

doi:10.1016/j.jaad.2005.07.052 
Table 1. Characteristics of included studies $(n=40)$

\begin{tabular}{|c|c|c|c|c|c|c|c|}
\hline Author, year & Location & $\begin{array}{l}\text { Study design, } \\
\text { LOE }\end{array}$ & Intervention & $\mathrm{N}^{\#}$ & $\begin{array}{c}\text { Mean age } \\
( \pm \mathrm{SD} / \mathrm{range} \\
)^{\prime}\end{array}$ & $F: M$ & Key findings \\
\hline $\begin{array}{l}\text { Abdel } \\
\text { Azim, } \\
2018\end{array}$ & Egypt & $\mathrm{RCT}^{\wedge}(1)$ & $\begin{array}{c}\mathrm{Nd}: Y A G \\
\mathrm{CO}_{2}+\mathrm{Nd}: Y \\
\mathrm{AG}\end{array}$ & 20 & $29.7(5)$ & $11: 9$ & $\begin{array}{l}\text { Higher satisfaction and lower } \\
\text { recurrence rates using } \\
\text { combined } \mathrm{CO}_{2} \text { and } \mathrm{Nd} \text { :YAG }\end{array}$ \\
\hline $\begin{array}{l}\text { Agut- } \\
\text { Busquet, } \\
2016\end{array}$ & Spain & $\begin{array}{c}\text { Case series } \\
\text { (3) }\end{array}$ & PDT & 7 & 28.9 & $3: 4$ & $\begin{array}{l}\text { Good results using PDT with } \\
\text { intralesional MB }\end{array}$ \\
\hline $\begin{array}{c}\text { Andino } \\
\text { Navarrete, } \\
2014\end{array}$ & Chile & $\begin{array}{c}\text { Case series } \\
\text { (3) }\end{array}$ & PDT & 5 & $26(5)$ & $4: 1$ & $\begin{array}{l}5-\mathrm{ALA} \text { and } 635 \mathrm{~nm} \text { light } \\
\text { improved quality of life and } \\
\text { reduced disease severity }\end{array}$ \\
\hline $\begin{array}{l}\text { Belotto, } \\
\text { 2013 }^{*}\end{array}$ & Brazil & $\begin{array}{l}\text { Case series } \\
\text { (3) }\end{array}$ & PDT & 5 & NS & $5: 0$ & $\begin{array}{l}\text { PDT improved inflammation } \\
\text { and drainage }\end{array}$ \\
\hline $\begin{array}{l}\text { Braunberg } \\
\text { er, } 2018^{*}\end{array}$ & USA & $\begin{array}{c}\text { Case series } \\
\text { (3) }\end{array}$ & $\mathrm{CO}_{2}$ & 38 & 37.5 & NS & $\begin{array}{l}\mathrm{CO} 2 \text { laser excision is a safe } \\
\text { and effective treatment }\end{array}$ \\
\hline $\mathrm{Bu}, 2017$ & China & $\begin{array}{c}\text { Case series } \\
\text { (3) }\end{array}$ & PDT & 7 & 24.4 & $0: 7$ & $\begin{array}{l}\text { Surgery combined with PDT } \\
\text { improved DLQI and VSS } \\
\text { scores }\end{array}$ \\
\hline $\begin{array}{l}\text { Calzavara- } \\
\text { Pinton, } \\
2013\end{array}$ & Italy & $\begin{array}{c}\text { Case series } \\
\text { (3) }\end{array}$ & PDT & 6 & 34 (10.9) & $5: 1$ & $\begin{array}{l}\text { MAL-PDT was effective and } \\
\text { well tolerated }\end{array}$ \\
\hline $\begin{array}{l}\text { Crocco, } \\
2015\end{array}$ & Brazil & $\begin{array}{c}\text { Case series } \\
\text { (3) }\end{array}$ & $\mathrm{CO}_{2}$ & 3 & 29.3 & $2: 1$ & $\begin{array}{l}\mathrm{CO}_{2} \text { laser effective in Hurley III } \\
\mathrm{HS}\end{array}$ \\
\hline $\begin{array}{c}\text { Darlymple, } \\
1987\end{array}$ & UK & $\begin{array}{c}\text { Case series } \\
\text { (3) }\end{array}$ & $\mathrm{CO}_{2}$ & 6 & NS & $5: 1$ & $\begin{array}{l}\mathrm{CO}_{2} \text { laser was effective with } \\
\text { minimal scarring }\end{array}$ \\
\hline $\begin{array}{l}\text { Esposito, } \\
2020\end{array}$ & Italy & $\begin{array}{c}\text { Case series } \\
\text { (3) }\end{array}$ & PEHT & 11 & 15.7 & $9: 2$ & $\begin{array}{l}\text { PEHT was effective with low } \\
\text { recurrence rates }\end{array}$ \\
\hline $\begin{array}{l}\text { Fabbrocini } \\
, 2018\end{array}$ & Italy & $\begin{array}{c}\text { Case series } \\
\text { (3) }\end{array}$ & $\begin{array}{l}\text { Diode } \\
\text { laser }\end{array}$ & 20 & $\begin{array}{l}26.6 \\
(7.84)\end{array}$ & $14: 6$ & $\begin{array}{l}1064 \mathrm{~nm} \text { intralesional diode } \\
\text { laser reduced Sartorius score } \\
\text { and DLQI, increased HiSCR }\end{array}$ \\
\hline $\begin{array}{l}\text { Fadel, } \\
2015\end{array}$ & Egypt & $\mathrm{RCT}^{\wedge}(1)$ & PDT & 10 & $\begin{array}{c}27.1 \\
(5.09)\end{array}$ & $7: 4$ & $\begin{array}{l}\text { Significant reduction in HS- } \\
\text { LASI with PDT }\end{array}$ \\
\hline $\begin{array}{l}\text { Finley, } \\
1996\end{array}$ & USA & $\begin{array}{c}\text { Case series } \\
\text { (3) }\end{array}$ & $\mathrm{CO}_{2}$ & 7 & $(20-46)$ & NS & $\begin{array}{l}\mathrm{CO}_{2} \text { laser is effective with low } \\
\text { recurrence rates }\end{array}$ \\
\hline $\begin{array}{l}\text { Giacaman, } \\
2019^{*}\end{array}$ & Spain & $\begin{array}{c}\text { Case series } \\
\text { (3) }\end{array}$ & PDT & 8 & NS & $43: 34$ & $\begin{array}{l}\text { Adalimumab and PDT } \\
\text { decreased lesions and pain }\end{array}$ \\
\hline Gold, 2004 & USA & $\begin{array}{c}\text { Case series } \\
\text { (3) }\end{array}$ & PDT & 4 & $\begin{array}{l}27.8(19- \\
46)\end{array}$ & $4: 0$ & $\begin{array}{l}\text { ALA-PDT is effective in HS } \\
\text { patients who did not respond } \\
\text { to medical therapy }\end{array}$ \\
\hline $\begin{array}{l}\text { Grimstad, } \\
2019\end{array}$ & Norway & $\begin{array}{c}\text { Case series } \\
\text { (2) }\end{array}$ & $\mathrm{CO}_{2}$ & 156 & $\begin{array}{l}39.3 \\
(9.91)\end{array}$ & $\begin{array}{c}191: 6 \\
7\end{array}$ & $\begin{array}{l}\text { Better response with surgical } \\
\text { versus medical treatments. } \\
\mathrm{CO}_{2} \text { laser was most effective. }\end{array}$ \\
\hline $\begin{array}{l}\text { Hazen, } \\
2010^{*}\end{array}$ & USA & $\begin{array}{c}\text { Case series } \\
\text { (3) }\end{array}$ & $\mathrm{CO}_{2}$ & 61 & $\begin{array}{l}38.5(21- \\
73)\end{array}$ & $42: 19$ & $\begin{array}{l}\mathrm{CO}_{2} \text { laser excision and } \\
\text { marsupialization is effective for } \\
\text { persistent or late-stage HS }\end{array}$ \\
\hline $\begin{array}{l}\text { Highton, } \\
2011\end{array}$ & UK & $\begin{array}{l}\text { Case series } \\
\text { (3) }\end{array}$ & IPL & 17 & $34(17-50)$ & $14: 3$ & $\begin{array}{l}\text { IPL reduced Sartorius scores } \\
\text { compared to control with good } \\
\text { patient satisfaction }\end{array}$ \\
\hline Jain, 2012 & India & $\begin{array}{c}\text { Case series } \\
\text { (3) }\end{array}$ & $\begin{array}{c}\mathrm{CO}_{2} \\
\mathrm{Nd}: Y A G\end{array}$ & 4 & $(30-40)$ & $4: 0$ & $\begin{array}{l}\text { Deroofing with } \mathrm{CO}_{2} \text { laser } \\
\text { combined with } \mathrm{Nd} \text { :YAG was } \\
\text { effective with no recurrence }\end{array}$ \\
\hline $\begin{array}{l}\text { Lapins, } \\
1994\end{array}$ & $\begin{array}{c}\text { Swede } \\
n\end{array}$ & $\begin{array}{c}\text { Case series } \\
\text { (3) }\end{array}$ & $\mathrm{CO}_{2}$ & 24 & $36(22-57)$ & $21: 3$ & $\begin{array}{l}\mathrm{CO}_{2} \text { laser was effective for } \\
\text { patients with chronic } \mathrm{HS} \text { with } \\
\text { low recurrence rates }\end{array}$ \\
\hline $\begin{array}{l}\text { Lapins, } \\
2002\end{array}$ & $\begin{array}{c}\text { Swede } \\
n\end{array}$ & $\begin{array}{l}\text { Case series } \\
\text { (3) }\end{array}$ & $\mathrm{CO}_{2}$ & 34 & $\begin{array}{c}33.9 \\
(11.0 / 15- \\
55)\end{array}$ & $31: 3$ & $\begin{array}{l}\text { Scanner-assisted } \mathrm{CO}_{2} \text { laser } \\
\text { ablation improved scarring and } \\
\text { had high patient satisfaction }\end{array}$ \\
\hline $\begin{array}{l}\text { Madan, } \\
2008\end{array}$ & UK & $\begin{array}{l}\text { Case series } \\
\text { (3) }\end{array}$ & $\mathrm{CO}_{2}$ & 9 & $39(27-52)$ & $8: 1$ & $\begin{array}{l}\mathrm{CO}_{2} \text { laser was an effective } \\
\text { treatment for patients with } \\
\text { recalcitrant HS }\end{array}$ \\
\hline
\end{tabular}

November $2021 \quad$ Volume 5 Issue 6 


\begin{tabular}{|c|c|c|c|c|c|c|c|}
\hline $\begin{array}{l}\text { Mahmoud, } \\
2010\end{array}$ & USA & $\operatorname{RCT}^{\wedge}(1)$ & Nd:YAG & 22 & $41(19-72)$ & 19:3 & $\begin{array}{l}\text { Nd:YAG + topical BPO \& } \\
\text { clindamycin better than topical } \\
\text { BPO \& clindamycin alone }\end{array}$ \\
\hline $\begin{array}{l}\text { Mikkelsen, } \\
2015\end{array}$ & $\begin{array}{c}\text { Denma } \\
\text { rk }\end{array}$ & $\begin{array}{c}\text { Case series } \\
\text { (3) }\end{array}$ & $\mathrm{CO}_{2}$ & 58 & $\begin{array}{l}37.8(21- \\
54)\end{array}$ & $48: 10$ & $\begin{array}{l}\mathrm{CO}_{2} \text { laser treatment effective } \\
\text { for recurrent } \mathrm{HS}\end{array}$ \\
\hline $\begin{array}{l}\text { Naouri, } \\
2020\end{array}$ & France & $\operatorname{RCT}^{\wedge}(1)$ & $\mathrm{Nd}: Y A G$ & 31 & NS & NS & $\begin{array}{l}\text { Nd:YAG laser for hair removal } \\
\text { has no effect on disease flares }\end{array}$ \\
\hline $\begin{array}{l}\text { Passeron, } \\
2009\end{array}$ & France & $\begin{array}{c}\text { Case series } \\
\text { (3) }\end{array}$ & PDT & 4 & NS & NS & $\begin{array}{l}\text { PDL-PDT was not effective in } \\
\text { treating HS }\end{array}$ \\
\hline $\begin{array}{l}\text { Piccolo, } \\
2014\end{array}$ & Italy & $\begin{array}{c}\text { Case series } \\
\text { (3) }\end{array}$ & IPL & 2 & $32(26-38)$ & $1: 1$ & $\begin{array}{l}\text { IPL completely resolved HS } \\
\text { lesions }\end{array}$ \\
\hline $\begin{array}{l}\text { Rodriguez } \\
\text {-Prieto, } \\
2013\end{array}$ & Spain & $\begin{array}{c}\text { Case series } \\
\text { (3) }\end{array}$ & PDT & 3 & $47(36-62)$ & $0: 3$ & No recurrence after ALA-PDT \\
\hline $\begin{array}{l}\text { Rucker } \\
\text { Wright, } \\
2009\end{array}$ & USA & $\begin{array}{l}\text { Case series } \\
\text { (3) }\end{array}$ & $\mathrm{Nd}: Y A G$ & 20 & NS & NS & $\begin{array}{l}\text { Nd:YAG laser reduced HS- } \\
\text { LASI scores }\end{array}$ \\
\hline $\begin{array}{l}\text { Shareef, } \\
2011\end{array}$ & UK & $\begin{array}{c}\text { Case series } \\
\text { (3) }\end{array}$ & PUVA & 13 & $35(25-66)$ & $11: 2$ & $\begin{array}{l}\text { PUVA is an effective treatment } \\
\text { in some patients }\end{array}$ \\
\hline $\begin{array}{l}\text { Sotiriou, } \\
2009\end{array}$ & Greece & $\begin{array}{c}\text { Case series } \\
\text { (3) }\end{array}$ & PDT & 5 & $\begin{array}{l}33.6(25- \\
43)\end{array}$ & $2: 3$ & $\begin{array}{l}\text { No significant improvement } \\
\text { after ALA-PDT }\end{array}$ \\
\hline $\begin{array}{l}\text { Strauss, } \\
2005\end{array}$ & UK & $\begin{array}{c}\text { Case series } \\
\text { (3) }\end{array}$ & PDT & $4^{\$}$ & NS & NS & $\begin{array}{l}\text { No significant improvement } \\
\text { after ALA-PDT }\end{array}$ \\
\hline $\begin{array}{c}\text { Suarez- } \\
\text { Valladares } \\
, 2018^{\star}\end{array}$ & Spain & $\begin{array}{c}\text { Case series } \\
\text { (3) }\end{array}$ & PDT & 5 & NS & NS & $\begin{array}{l}\text { Resolution with no recurrence } \\
\text { after ALA-PDT }\end{array}$ \\
\hline $\begin{array}{l}\text { Suarez- } \\
\text { Valladares } \\
, 2017\end{array}$ & Spain & $\begin{array}{c}\text { Case series } \\
\text { (3) }\end{array}$ & PDT & 38 & $36(30-44)$ & $18: 20$ & $\begin{array}{l}\text { Complete response in most } \\
\text { patients after ALA-PDT }\end{array}$ \\
\hline $\begin{array}{l}\text { Theut Riis, } \\
2018\end{array}$ & $\begin{array}{l}\text { Denma } \\
\text { rk }\end{array}$ & $\begin{array}{l}\text { Case series } \\
\text { (3) }\end{array}$ & IPL & 25 & $\begin{array}{c}39.2 \\
(10.9 / 17- \\
63)\end{array}$ & $25: 0$ & $\begin{array}{l}\text { IPL effective as an adjuvant } \\
\text { treatment in a subset of } \\
\text { patients }\end{array}$ \\
\hline $\begin{array}{l}\text { Valladares } \\
\text {-Narganes } \\
\text {, } 2015\end{array}$ & Spain & $\begin{array}{c}\text { Case series } \\
\text { (3) }\end{array}$ & PDT & 27 & $\begin{array}{l}30.3(19- \\
62)\end{array}$ & $11: 16$ & $\begin{array}{l}\text { ALA-PDT effective in most } \\
\text { patients and improved } \\
\text { Sartorius score }\end{array}$ \\
\hline $\begin{array}{c}\text { Vilarrasa, } \\
2019^{*}\end{array}$ & Spain & $\begin{array}{c}\text { Case series } \\
\text { (3) }\end{array}$ & PDT & 28 & 46 & $13: 15$ & $\begin{array}{l}\text { ALA-PDT improved DLQI and } \\
\text { pain }\end{array}$ \\
\hline $\begin{array}{l}\text { Vossen, } \\
2018\end{array}$ & $\begin{array}{l}\text { The } \\
\text { Netherl } \\
\text { ands }\end{array}$ & $\begin{array}{c}\text { Case series } \\
\text { (3) }\end{array}$ & Nd:YAG & 15 & $\begin{array}{c}34.1 \\
(10.1)\end{array}$ & $5: 10$ & $\begin{array}{l}\text { Nd:YAG reduced number of } \\
\text { flares and reduced disease } \\
\text { severity }\end{array}$ \\
\hline $\begin{array}{l}\text { Wilden, } \\
2019\end{array}$ & $\begin{array}{c}\text { Germa } \\
\text { ny }\end{array}$ & RCT (1) & $\begin{array}{l}\mathrm{IPL}+ \\
\text { radiofrequ } \\
\text { ency }\end{array}$ & 41 & $38(23-57)$ & $31: 12$ & $\begin{array}{l}\text { IPL and radiofrequency } \\
\text { reduced lesion count and DLQ } \\
\text { compared to either treatment } \\
\text { alone }\end{array}$ \\
\hline $\mathrm{Xu}, 2011$ & USA & $\operatorname{RCT}^{\wedge}(1)$ & Nd:YAG & 19 & $37(23-54)$ & $16: 3$ & $\begin{array}{l}\text { Nd:YAG improved HS-LASI } \\
\text { score }\end{array}$ \\
\hline $\begin{array}{l}\text { Zhang, } \\
2016\end{array}$ & China & $\begin{array}{c}\text { Case series } \\
\text { (3) }\end{array}$ & PDT & 3 & $(17-38)$ & $0: 3$ & $\begin{array}{l}\text { ALA-PDT ineffective for late- } \\
\text { stage HS }\end{array}$ \\
\hline
\end{tabular}

ALA: aminolevulinic acid, BPO: benzoyl peroxide, $\mathbf{C O}_{2}$ : carbon dioxide, DLQI: Dermatology Life Quality Index, F: female, HiSCR: Hidradenitis Suppurativa Clinical Response, HS: hidradenitis suppurativa, HS-LASI: Hidradenitis Suppurativa Lesion, Area, and Severity Index, IPL: intense pulsed light, LOE: Level of evidence, MAL: methyl aminolevulinate, M: male, Nd:YAG: neodymium-doped yttrium aluminum garnet, NS: not specified, PDL: pulsed dye laser, PDT: photodynamic therapy, PEHT: pediatric endoscopic HS treatment, PUVA: psoralen and ultraviolet A, RCT: randomized controlled trial

"Abstract only

\# Number of participants included in analysis

$\$ 2$ participants completed the study

${ }^{\wedge}$ Randomized within-patient controlled trial

Key to evidence-based support: (1) prospective controlled trial; (2) retrospective study or large case series; (3) small case series or individual case reports 
Table 2. Summary of treatment outcomes by phototherapy intervention for HS

\begin{tabular}{|c|c|c|}
\hline Light and Laser-based Treatment Modality (pooled n) & $\begin{array}{c}\% \text { Patients with Any } \\
\text { Response (n) }\end{array}$ & $\begin{array}{c}\% \text { Adverse Events } \\
(\mathrm{n})\end{array}$ \\
\hline 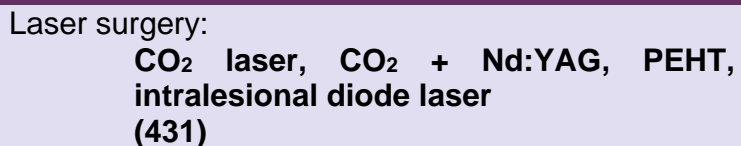 & $80(344)$ & $25(105 / 424)$ \\
\hline $\begin{array}{l}\text { CO2 } \\
(396)\end{array}$ & $79(311)$ & $26(103 / 393)$ \\
\hline $\begin{array}{l}\text { CO2 + Nd:YAG } \\
\text { (4) }\end{array}$ & $100(4)$ & NR \\
\hline $\begin{array}{l}\text { PEHT } \\
\text { (11) }\end{array}$ & $100(11)$ & $0(0 / 11)$ \\
\hline $\begin{array}{l}\text { Intralesional diode laser } \\
(20)\end{array}$ & $90(18)$ & $10(2 / 20)$ \\
\hline $\begin{array}{l}\text { Field treatment: } \\
\text { Nd:YAG laser, IPL, diode } \\
\text { (101) }\end{array}$ & $71(84)$ & $18(24 / 134)$ \\
\hline $\begin{array}{l}\text { PDT } \\
\text { (169) }\end{array}$ & $73(124)$ & $36(23 / 64)$ \\
\hline $\begin{array}{l}\text { PUVA } \\
\text { (13) }\end{array}$ & $69(9)$ & $15(2 / 13)$ \\
\hline
\end{tabular}

$\mathrm{n}$ - number of patients with outcomes reported

CO2: carbon dioxide, IPL: intense pulsed light, Nd:YAG: neodymium-doped yttrium aluminum garnet, PDL: pulsed dye laser, PDT: photodynamic therapy, PEHT: pediatric endoscopic HS treatment, PUVA: psoralen and ultraviolet A 
Table 3. Results of included studies examining multiple outcome measures on hidradenitis suppurativa

\begin{tabular}{|c|c|c|c|c|c|c|c|c|}
\hline $\begin{array}{l}\text { Author } \\
\text {, Year }\end{array}$ & $\mathrm{N}$ & $\begin{array}{l}\text { Mean } \\
\text { age } \\
\text { ( } \pm S D / \\
\text { range) }\end{array}$ & Intervention & $\begin{array}{l}\text { Outcome } \\
\text { measure }\end{array}$ & $\begin{array}{l}\text { Mean before } \\
( \pm S D)\end{array}$ & $\begin{array}{l}\text { Mean after } \\
( \pm S D)\end{array}$ & Delta & Conclusion \\
\hline $\begin{array}{l}\text { Abdel } \\
\text { Azim, } \\
2018\end{array}$ & 20 & $\begin{array}{l}29.7 \\
( \pm 5)\end{array}$ & $\begin{array}{l}\mathrm{Nd}: Y A G \text { and } \\
\text { combined } \\
\mathrm{CO}_{2} / \mathrm{Nd}: \mathrm{YA} \\
\mathrm{G}\end{array}$ & $\begin{array}{l}\text { VAS patient } \\
\text { satisfaction }\end{array}$ & $\begin{array}{l}\mathrm{CO}_{2} / \mathrm{Nd}: Y A G: \\
20.6 \\
\mathrm{Nd}: Y A G: 20.4 \\
\mathrm{~N} / \mathrm{A}\end{array}$ & $\begin{array}{l}\mathrm{CO}_{2} / \mathrm{Nd}: Y A G: \\
16.9 \\
\mathrm{Nd}: Y A G: 24.1 \\
\mathrm{CO}_{2} / \mathrm{Nd}: Y A G: \\
24 ; \mathrm{Nd}: Y A G: 17\end{array}$ & $\begin{array}{l}\mathrm{CO}_{2} / \mathrm{Nd}: Y A G: \\
-3.7 \\
\mathrm{Nd}: Y A G: 3.7 \\
\mathrm{~N} / \mathrm{A}\end{array}$ & \multirow{2}{*}{$\begin{array}{l}\mathrm{CO}_{2}+\mathrm{Nd}: \mathrm{YAG} \text { superior to } \\
\mathrm{Nd}: \mathrm{YAG} \text { alone. Combined } \\
\text { treatment had a lower rate of } \\
\text { recurrence and higher patient } \\
\text { satisfaction. } \\
\text { I-PDT is more effective for HS } \\
\text { Hurley stage II than stage III. } \\
\text { At } 6 \text { months, } 5 / 7 \text { patients had } \\
\text { no recurrence. }\end{array}$} \\
\hline $\begin{array}{l}\text { Agut- } \\
\text { Busq } \\
\text { uet, } \\
2018\end{array}$ & 7 & 28.9 & I-PDT & $\begin{array}{l}\text { VAS } \\
\text { DLQI } \\
\text { PGA } \\
\text { US } \\
\text { assessments }\end{array}$ & $\begin{array}{l}4.6( \pm 1.4) \\
9.4( \pm 3.9) \\
3( \pm 2.2) \\
\text { Large } \\
\text { transverse } \\
\text { diameter }\end{array}$ & $\begin{array}{l}\text { N/A } \\
2.2( \pm 2.1) \\
\text { N/A } \\
\text { Reduced } \\
\text { diameter in } \\
\text { treated lesions }\end{array}$ & $\begin{array}{l}\text { N/A } \\
-7.2 \\
N / A \\
N / A\end{array}$ & \\
\hline $\begin{array}{l}\text { Andin } \\
\text { o } \\
\text { Navar } \\
\text { rete, } \\
2014\end{array}$ & 5 & $\begin{array}{l}26 \\
( \pm 5)\end{array}$ & $\begin{array}{l}\text { PDT (5- } \\
\text { ALA; } 635 \mathrm{~nm} \\
\text { light) }\end{array}$ & $\begin{array}{l}\text { Sartorius } \\
\text { DLQI } \\
\text { VAS }\end{array}$ & $\begin{array}{l}35.4( \pm 4.98) \\
28.8( \pm 2.68) \\
3( \pm 0)\end{array}$ & $\begin{array}{l}18.2( \pm 8.11) \\
7.49( \pm 2.79) \\
0.8( \pm 0.45)\end{array}$ & $\begin{array}{l}-17.2 \\
-21.31 \\
-2.2\end{array}$ & $\begin{array}{l}\text { PDT improved QOL and } \\
\text { reduced severity of disease. }\end{array}$ \\
\hline $\begin{array}{l}\text { Belott } \\
0, \\
2013\end{array}$ & 5 & $\mathrm{~N} / \mathrm{A}$ & $\mathrm{PDT}^{\#}$ & $\begin{array}{l}\text { Inflammation, } \\
\text { drainage, } \\
\text { erythema, } \\
\text { edema }\end{array}$ & $\mathrm{N} / \mathrm{A}$ & $\begin{array}{l}\text { Reduced } \\
\text { drainage and } \\
\text { inflammation in } \\
60 \% \text {; partial } \\
\text { reduction in } \\
40 \% \text {. }\end{array}$ & $\mathrm{N} / \mathrm{A}$ & $\begin{array}{l}\text { PDT improves inflammation } \\
\text { and drainage }\end{array}$ \\
\hline \multirow[t]{3}{*}{$\begin{array}{l}\text { Braun } \\
\text { berge } \\
r, \\
2018\end{array}$} & 38 & 37.5 & $\mathrm{CO}_{2}$ laser & $\begin{array}{l}\text { Healing time in } \\
\text { smokers }\end{array}$ & $N / A$ & $\begin{array}{l}\text { Smokers: } 6 \\
\text { months } \\
\text { Non-smokers: } 6 \\
\text { months }\end{array}$ & $\mathrm{N} / \mathrm{A}$ & \multirow{3}{*}{$\begin{array}{l}\text { After } \mathrm{CO}_{2} \text { excision - smoking } \\
\text { did not affect healing time but } \\
\text { diabetics had a prolonged } \\
\text { healing time. } \\
3 \text { patients experienced } \\
\text { recurrence at a mean of } 6 \\
\text { months post procedure. }\end{array}$} \\
\hline & & & & $\begin{array}{l}\text { Healing time in } \\
\text { diabetics }\end{array}$ & $\mathrm{N} / \mathrm{A}$ & $\begin{array}{l}\text { Diabetics: } 7.3 \\
\text { months } \\
\text { Non-diabetics: } \\
5.4 \text { months }\end{array}$ & $\mathrm{N} / \mathrm{A}$ & \\
\hline & & & & Recurrence & $\mathrm{N} / \mathrm{A}$ & 3 patients & $\mathrm{N} / \mathrm{A}$ & \\
\hline \multirow{3}{*}{$\begin{array}{l}\text { Bu, } \\
2017\end{array}$} & 7 & 24.4 & Surgery & Healing time & $\mathrm{N} / \mathrm{A}$ & 29.4 days & $\mathrm{N} / \mathrm{A}$ & \multirow{3}{*}{$\begin{array}{l}\text { Surgery in combination with } \\
\text { PDT improved DLQI. No } \\
\text { recurrence. Advantage of } \\
\text { faster healing and less } \\
\text { scaring. }\end{array}$} \\
\hline & & & $\begin{array}{l}\text { combined } \\
\text { with PDT }\end{array}$ & $\begin{array}{l}\text { Recurrence } \\
\text { DLQI }\end{array}$ & $\begin{array}{l}\mathrm{N} / \mathrm{A} \\
24.1( \pm 4.3)\end{array}$ & $\begin{array}{l}\text { No recurrence } \\
5 \text { months post } \\
\text { treatment: } 4.9( \pm \\
2.8)\end{array}$ & $\begin{array}{l}N / A \\
-19.2\end{array}$ & \\
\hline & & & & VSS & $\mathrm{N} / \mathrm{A}$ & $4.6( \pm 2.4)$ & $\mathrm{N} / \mathrm{A}$ & \\
\hline \multirow{3}{*}{$\begin{array}{l}\text { Calza } \\
\text { vara- } \\
\text { Pinto } \\
\text { n, } \\
2013\end{array}$} & 6 & $\begin{array}{l}34( \pm \\
10.9)\end{array}$ & MAL-PDT & $\begin{array}{l}\text { Clinical } \\
\text { response }\end{array}$ & $\mathrm{N} / \mathrm{A}$ & $\begin{array}{l}\text { No/poor } \\
\text { response: } 17 \% \text {; } \\
\text { Moderate: } 50 \% \text {; } \\
\text { Marked: } 33 \%\end{array}$ & $\mathrm{~N} / \mathrm{A}$ & \multirow{3}{*}{$\begin{array}{l}2 / 6 \text { patients had marked } \\
\text { improvement, } 3 \text { had moderate } \\
\text { improvement, and } 1 \text { had } \\
\text { no/poor response with MAL- } \\
\text { PDT treatment. }\end{array}$} \\
\hline & & & & Local reaction & $N / A$ & $\begin{array}{l}\text { Absent: } 17 \% \text {; } \\
\text { Moderate: } 83 \% \text {; } \\
\text { Marked: } 0 \%\end{array}$ & $\mathrm{~N} / \mathrm{A}$ & \\
\hline & & & & Cosmesis & $\mathrm{N} / \mathrm{A}$ & $33 \%$ & $\mathrm{~N} / \mathrm{A}$ & \\
\hline $\begin{array}{l}\text { Crocc } \\
0 \\
2015\end{array}$ & 3 & 29.3 & $\mathrm{CO}_{2}$ laser & $\begin{array}{l}\text { Clinical } \\
\text { response }\end{array}$ & $\begin{array}{l}\text { Patient 1: } \\
\text { abscesses in } \\
\text { axilla } \\
\text { Patient 2: } \\
\text { abscesses in } \\
\text { axillary, } \\
\text { inframammary } \\
\text { and inguinal } \\
\text { regions } \\
\text { Patient 3: } \\
\text { abscesses in } \\
\text { axilla \& groin }\end{array}$ & $\begin{array}{l}\text { Patient 1: } \\
\text { successful } \\
\text { treatment } \\
\text { Patient 2: } \\
\text { successful } \\
\text { treatment } \\
\text { Patient 3: } \\
\text { successful } \\
\text { treatment }\end{array}$ & $\mathrm{N} / \mathrm{A}$ & $\begin{array}{l}\text { CO2 laser effective in Hurley } \\
\text { III HS. }\end{array}$ \\
\hline \multirow{2}{*}{$\begin{array}{l}\text { Darly } \\
\text { mple, } \\
1987\end{array}$} & 6 & $\begin{array}{l}(20- \\
43)\end{array}$ & $\mathrm{CO}_{2}$ laser & $\begin{array}{l}\text { Wound } \\
\text { healing }\end{array}$ & $\mathrm{N} / \mathrm{A}$ & 3-7 weeks & $\mathrm{N} / \mathrm{A}$ & \multirow{2}{*}{$\begin{array}{l}\text { Patients reported limited } \\
\text { scarring. All patients were } \\
\text { disease free at } 9 \text { months to } 3 \\
\text { years follow up. All }\end{array}$} \\
\hline & & & & Recurrence & $\mathrm{N} / \mathrm{A}$ & None & $\mathrm{N} / \mathrm{A}$ & \\
\hline
\end{tabular}




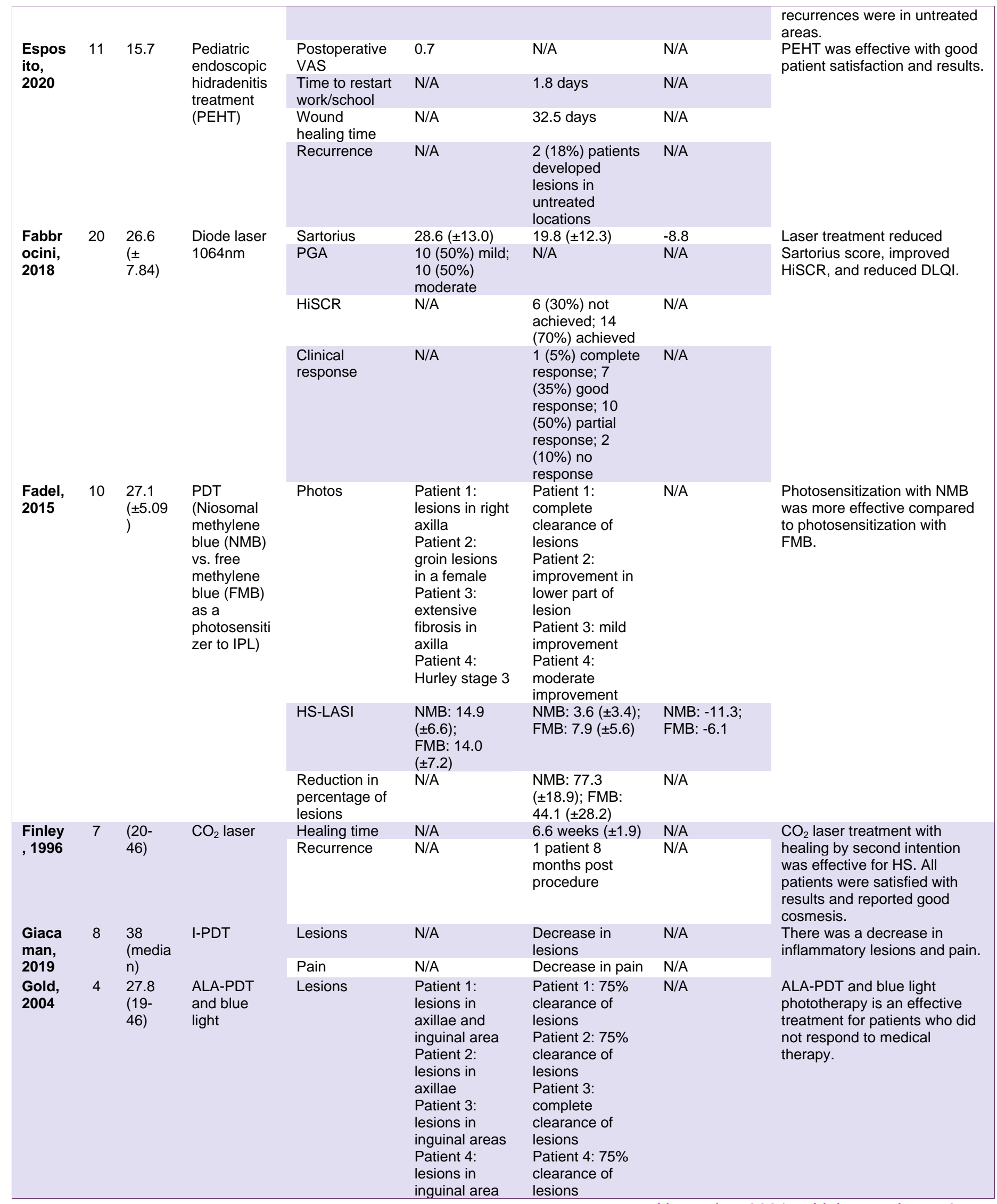

November $2021 \quad$ Volume 5 Issue 6 


\begin{tabular}{|c|c|c|c|c|c|c|c|c|}
\hline $\begin{array}{l}\text { Grims } \\
\text { tad, } \\
2019\end{array}$ & $\begin{array}{c}15 \\
6\end{array}$ & $\begin{array}{l}39.3 \\
( \pm 9.91 \\
)^{3}\end{array}$ & $\begin{array}{l}\mathrm{CO}_{2} \text { laser, } \\
\mathrm{CO}_{2} \text { laser } \\
\text { and topical } \\
\text { clindamycin, } \\
\mathrm{CO}_{2} \text { laser } \\
\text { and } \\
\text { systemic } \\
\text { antibiotic }\end{array}$ & $\begin{array}{l}\text { Sartorius } \\
\text { score }\end{array}$ & $\begin{array}{l}\mathrm{CO}_{2} \text { laser } \\
(\mathrm{n}=156): 57 ; \\
\mathrm{CO}_{2} \text { laser and } \\
\text { topical } \\
\text { clindamycin } \\
(\mathrm{n}=5): 85 ; \\
\mathrm{CO}_{2} \text { laser and } \\
\text { systemic } \\
\text { antibiotic } \\
(\mathrm{n}=1): 123\end{array}$ & $\mathrm{~N} / \mathrm{A}$ & $\begin{array}{l}\mathrm{CO}_{2} \text { laser }(\mathrm{n} \\
=156):-33 ; \\
\mathrm{CO}_{2} \text { laser and } \\
\text { topical } \\
\text { clindamycin } \\
(\mathrm{n}=5):-36 ; \\
\mathrm{CO}_{2} \text { laser and } \\
\text { systemic } \\
\text { antibiotic ( } \mathrm{n} \\
=1):-87\end{array}$ & $\begin{array}{l}\mathrm{CO}_{2} \text { laser was the most } \\
\text { effective intervention studied. } \\
\text { Almost half of patients treated } \\
\text { with a combination of surgical } \\
\text { and medical treatments had a } \\
\text { significant improvement in } \\
\text { Sartorius score. }\end{array}$ \\
\hline & & & & DLQI & $\begin{array}{l}\mathrm{CO}_{2} \text { laser } \\
(\mathrm{n}=156): 14 ; \\
\mathrm{CO}_{2} \text { laser and } \\
\text { topical } \\
\text { clindamycin } \\
(\mathrm{n}=5): 14 ; \\
\mathrm{CO}_{2} \text { laser and } \\
\text { systemic } \\
\text { antibiotic } \\
(\mathrm{n}=1): 13\end{array}$ & $\mathrm{~N} / \mathrm{A}$ & $\begin{array}{l}\mathrm{CO}_{2} \text { laser }(\mathrm{n} \\
=156):-5 ; \mathrm{CO}_{2} \\
\text { laser and } \\
\text { topical } \\
\text { clindamycin } \\
(\mathrm{n}=5):-6 ; \mathrm{CO}_{2} \\
\text { laser and } \\
\text { systemic } \\
\text { antibiotic ( } \mathrm{n} \\
=1): 0\end{array}$ & \\
\hline \multirow[t]{3}{*}{$\begin{array}{l}\text { Hazen } \\
, 2010\end{array}$} & 61 & $\begin{array}{l}38.5 \\
(21- \\
73)\end{array}$ & $\begin{array}{l}\mathrm{CO}_{2} \text { laser } \\
\text { excision and } \\
\text { marsupializa } \\
\text { tion }\end{array}$ & $\begin{array}{l}\text { Recurrence of } \\
\text { disease }\end{array}$ & $\mathrm{N} / \mathrm{A}$ & $\begin{array}{l}\text { Recurrence in } 2 \\
\text { patients. } \\
\text { Average of } 4.1 \\
\text { years without } \\
\text { recurrence in } \\
\text { treated areas } \\
\text { (range of } 1-17 \\
\text { years). }\end{array}$ & $\mathrm{N} / \mathrm{A}$ & \multirow[t]{3}{*}{$\begin{array}{l}\mathrm{CO}_{2} \text { laser excision \& } \\
\text { marsupialization effective } \\
\text { therapy for persistent or late- } \\
\text { stage } \mathrm{HS} \text { (when scarring and } \\
\text { sinus tract formation present). }\end{array}$} \\
\hline & & & & $\begin{array}{l}\text { Comfort/ } \\
\text { patient } \\
\text { satisfaction }\end{array}$ & $\mathrm{N} / \mathrm{A}$ & $\begin{array}{l}\text { Treatments well } \\
\text { tolerated in all } \\
\text { patients. }\end{array}$ & $\mathrm{N} / \mathrm{A}$ & \\
\hline & & & & Healing time & $\mathrm{N} / \mathrm{A}$ & 8.8 weeks & $\mathrm{N} / \mathrm{A}$ & \\
\hline \multirow[t]{3}{*}{$\begin{array}{l}\text { Hight } \\
\text { on, } \\
2011\end{array}$} & 17 & $\begin{array}{l}34 \\
(17- \\
50)\end{array}$ & $\begin{array}{l}\text { IPL } \\
\text { (Harmony } \\
\text { laser; } \\
\text { 2x/week for } \\
4 \text { weeks on } \\
\text { one area vs } \\
\text { contralateral } \\
\text { side not } \\
\text { treated - } \\
\text { served as } \\
\text { control) }\end{array}$ & $\begin{array}{l}\text { Sartorius } \\
\text { score }\end{array}$ & $\mathrm{N} / \mathrm{A}$ & $\begin{array}{l}3 \text { months post } \\
\text { treatment: }-56 \% \text {. } \\
6 \text { months: }-44 \% \text {. } \\
12 \text { months: - } \\
33 \% \text {. } \\
\text { Control side - } \\
3 \text { months: }-10 \% \text {. } \\
6 \text { months: }-10 \% \text {. } \\
12 \text { months: } 3 \% \text {. }\end{array}$ & $\begin{array}{l}\text { Significant } \\
\text { reduction in } \\
\text { HS exam } \\
\text { score on } \\
\text { treated side } \\
(p<0.001) . \\
\text { Significant } \\
\text { difference } \\
\text { between } \\
\text { treated and } \\
\text { control sides } \\
(p<0.001) .\end{array}$ & \multirow[t]{3}{*}{$\begin{array}{l}\text { Reduction in severity of HS } \\
\text { with IPL. Mean examination } \\
\text { score was improved and } \\
\text { maintained at } 12 \text { months. } \\
\text { Improvement was also } \\
\text { reported by independent } \\
\text { analysis of clinical } \\
\text { photographs. Patients } \\
\text { reported high levels of } \\
\text { satisfaction with treatment. } \\
\text { Treated side had significant } \\
\text { reduction in Sartorius score } \\
\text { maintained at } 12 \text { months } \\
(p=0.001) \text {. }\end{array}$} \\
\hline & & & & $\begin{array}{l}\text { Patient } \\
\text { satisfaction }\end{array}$ & $\mathrm{N} / \mathrm{A}$ & $\begin{array}{l}\text { Treatment side } \\
\text { Disease } \\
\text { clearance: } 1 \\
\text { patient } \\
\text { Excellent } \\
\text { results: } 2 \\
\text { patients } \\
\text { Good results: } 10 \\
\text { patients } \\
4 \text { patients: fair } \\
\text { results }\end{array}$ & $\mathrm{N} / \mathrm{A}$ & \\
\hline & & & & & & $\begin{array}{l}\text { Control side: } \\
\text { No change: } 15 \\
\text { patients } \\
\text { Slight } \\
\text { improvement: } 1 \\
\text { patient } \\
\text { Slight decline: } 1 \\
\text { patient }\end{array}$ & & \\
\hline $\begin{array}{l}\text { Jain, } \\
2012\end{array}$ & 4 & $\begin{array}{l}(30- \\
40)\end{array}$ & $\begin{array}{l}\mathrm{Nd}: Y A G \text { and } \\
\text { CO2 laser }\end{array}$ & $\begin{array}{l}\text { Recurrence of } \\
\text { disease }\end{array}$ & $N / A$ & No recurrence & $\mathrm{N} / \mathrm{A}$ & $\begin{array}{l}\text { Deroofing with } \mathrm{CO}_{2} \text { laser } \\
\text { combined with } \mathrm{Nd}: Y A G \text { laser } \\
\text { is effective in treating } \mathrm{HS} \text {, with } \\
\text { no recurrence observed up to } \\
3 \text { years. }\end{array}$ \\
\hline
\end{tabular}




\begin{tabular}{|c|c|c|c|c|c|c|c|c|}
\hline \multirow[t]{4}{*}{$\begin{array}{l}\text { Lapin } \\
\text { s, } \\
1994\end{array}$} & \multirow[t]{4}{*}{24} & \multirow[t]{4}{*}{$\begin{array}{l}36 \\
(22- \\
57)\end{array}$} & $\begin{array}{l}\mathrm{CO}_{2} \text { laser } \\
\text { with healing } \\
\text { by }\end{array}$ & $\begin{array}{l}\text { Recurrence of } \\
\text { disease }\end{array}$ & $\mathrm{N} / \mathrm{A}$ & $\begin{array}{l}\text { Recurrence: } 2 \\
\text { No recurrence: } \\
22\end{array}$ & $\mathrm{~N} / \mathrm{A}$ & \multirow{4}{*}{$\begin{array}{l}\mathrm{CO}_{2} \text { laser treatment with } \\
\text { secondary intention for } \\
\text { patients with chronic HS is } \\
\text { beneficial, safe, and quick, } \\
\text { leaving patients with good } \\
\text { cosmetic and functional } \\
\text { results. }\end{array}$} \\
\hline & & & \multirow[t]{3}{*}{$\begin{array}{l}\text { secondary } \\
\text { intention }\end{array}$} & Healing time & $\mathrm{N} / \mathrm{A}$ & $\begin{array}{l}4 \text { weeks }(3-5 \\
\text { weeks) }\end{array}$ & $\mathrm{N} / \mathrm{A}$ & \\
\hline & & & & $\begin{array}{l}\text { Scarring/cosm } \\
\text { etic } \\
\text { appearance }\end{array}$ & $\mathrm{N} / \mathrm{A}$ & $\begin{array}{l}\text { All patients } \\
\text { satisfied with } \\
\text { scar } \\
\text { appearance. }\end{array}$ & $\mathrm{N} / \mathrm{A}$ & \\
\hline & & & & $\begin{array}{l}\text { Patient } \\
\text { satisfaction }\end{array}$ & $\mathrm{N} / \mathrm{A}$ & $\begin{array}{l}\text { Patients found } \\
\text { this method } \\
\text { favourable, } \\
\text { willing to repeat } \\
\text { procedure if } \\
\text { required. }\end{array}$ & $\mathrm{N} / \mathrm{A}$ & \\
\hline \multirow[t]{4}{*}{$\begin{array}{l}\text { Lapin } \\
\text { s, } \\
2002\end{array}$} & \multirow[t]{4}{*}{34} & \multirow[t]{4}{*}{$\begin{array}{l}( \pm 11.0 \\
/ 15- \\
55)\end{array}$} & \multirow{4}{*}{$\begin{array}{l}\text { Scanner- } \\
\text { assisted } \\
\mathrm{CO}_{2} \text { laser } \\
\text { ablation }\end{array}$} & Recurrence & $\mathrm{N} / \mathrm{A}$ & $\begin{array}{l}\text { Recurrence: } 4 \\
\text { No recurrence: } \\
30\end{array}$ & $\mathrm{~N} / \mathrm{A}$ & \multirow{4}{*}{$\begin{array}{l}\mathrm{CO}_{2} \text { laser with scanner is a } \\
\text { fast and efficient treatment of } \\
\mathrm{HS} \text {, with satisfactory cosmetic } \\
\text { and functional results. }\end{array}$} \\
\hline & & & & Healing time & $\mathrm{N} / \mathrm{A}$ & $\begin{array}{l}4 \text { weeks (3-5 } \\
\text { weeks) }\end{array}$ & $\mathrm{N} / \mathrm{A}$ & \\
\hline & & & & $\begin{array}{l}\text { Pain in post- } \\
\text { op period } \\
\text { (Scale from 0- } \\
\text { 3) }\end{array}$ & $\mathrm{N} / \mathrm{A}$ & $\begin{array}{l}\text { Classified as } 3: \\
4 \\
\text { Classified as } 2: \\
15 \\
\text { Classified as } 1: \\
9 \\
\text { Classified as } 0: \\
6\end{array}$ & $\mathrm{~N} / \mathrm{A}$ & \\
\hline & & & & $\begin{array}{l}\text { Patient } \\
\text { satisfaction }\end{array}$ & $\mathrm{N} / \mathrm{A}$ & $\begin{array}{l}\text { Condition better } \\
\text { than pre } \\
\text { surgery: } 31 \\
\text { patients } \\
\text { Hardly changed: } \\
2 \text { patients } \\
\text { Worse: } 1 \text { patient }\end{array}$ & $\mathrm{N} / \mathrm{A}$ & \\
\hline \multirow[t]{3}{*}{$\begin{array}{l}\text { Mada } \\
\text { n, } \\
2008\end{array}$} & \multirow[t]{3}{*}{9} & \multirow[t]{3}{*}{$\begin{array}{l}39 \\
(27- \\
52)\end{array}$} & \multirow{3}{*}{$\begin{array}{l}\mathrm{CO}_{2} \text { laser } \\
\text { excision } \\
\text { (prophylactic } \\
\text { oral } \\
\text { antibiotics } \\
\text { for } 2 \text { weeks } \\
\text { post-op) }\end{array}$} & $\begin{array}{l}\text { Recurrence of } \\
\text { disease }\end{array}$ & $\mathrm{N} / \mathrm{A}$ & $\begin{array}{l}\text { No recurrence: } 6 \\
\text { Active HS at } \\
\text { untreated sites } \\
\text { adjacent to } \\
\text { treated sites: } 2\end{array}$ & $\mathrm{~N} / \mathrm{A}$ & \multirow[t]{3}{*}{$\begin{array}{l}\mathrm{CO}_{2} \text { laser is an effective } \\
\text { treatment for patients with } \\
\text { recalcitrant HS, with patients } \\
\text { reporting high satisfaction } \\
\text { scores. }\end{array}$} \\
\hline & & & & $\begin{array}{l}\text { Patient } \\
\text { satisfaction }\end{array}$ & $\mathrm{N} / \mathrm{A}$ & $\begin{array}{l}8.5 / 10 \text { (range of } \\
7-10)\end{array}$ & $\mathrm{N} / \mathrm{A}$ & \\
\hline & & & & $\begin{array}{l}\text { Wound } \\
\text { healing time }\end{array}$ & $\mathrm{N} / \mathrm{A}$ & $\begin{array}{l}2 \text { weeks (range } \\
\text { of } 1-4 \text { ) }\end{array}$ & $\mathrm{N} / \mathrm{A}$ & \\
\hline \multirow[t]{3}{*}{$\begin{array}{l}\text { Mahm } \\
\text { oud, } \\
2010\end{array}$} & \multirow[t]{3}{*}{22} & \multirow[t]{3}{*}{$\begin{array}{l}41 \\
(19- \\
72)\end{array}$} & \multirow{3}{*}{$\begin{array}{l}\text { Nd:YAG } \\
\text { laser } \\
\text { (combined } \\
\text { with topical } \\
\text { benzoyl } \\
\text { peroxide } \\
\text { wash and } \\
\text { clindamycin } \\
\text { lotion; } \\
\text { benzoyl } \\
\text { peroxide } \\
\text { and } \\
\text { clindamycin } \\
\text { alone used } \\
\text { as control) }\end{array}$} & $\begin{array}{l}\text { Modified } \\
\text { Sartorius } \\
\text { score (HS } \\
\text { Lesion Area } \\
\text { and Severity } \\
\text { Index) }\end{array}$ & $\begin{array}{l}\text { At baseline for } \\
\text { all sites } \\
\text { combined: } 31 \\
\text { (SD: } 14.9 \text { ) } \\
\text { All control } \\
\text { sites } \\
\text { combined: } 29 \\
\text { (SD:13.2) }\end{array}$ & $\begin{array}{l}\text { At } 6 \text { months } \\
\text { Treated sites: } \\
7.6 \text { (SD: } 4.1 \text { ) } \\
\text { Mean for all } \\
\text { control sites: } \\
19.9 \text { (SD: } 10.9)\end{array}$ & $\begin{array}{l}\text { Average } \\
\text { improvement } \\
\text { over all } \\
\text { anatomic sites } \\
\text { was } 72.7 \% \text { on } \\
\text { laser treated } \\
\text { side and } \\
22.9 \% \text { on } \\
\text { control side } \\
(p<0.05) .\end{array}$ & \multirow[t]{3}{*}{$\begin{array}{l}\text { Nd:YAG laser \& topical } \\
\text { benzoyl peroxide + } \\
\text { clindamycin are associated } \\
\text { with progressive improvement } \\
\text { of HS lesions, and are more } \\
\text { effective than topical benzoyl } \\
\text { peroxide + clindamycin alone. } \\
\text { Overall high level of patient } \\
\text { satisfaction with treatment. }\end{array}$} \\
\hline & & & & $\begin{array}{l}\text { Patient } \\
\text { satisfaction - } \\
\text { Pain } \\
\text { associated } \\
\text { with HS }\end{array}$ & $\mathrm{N} / \mathrm{A}$ & $\begin{array}{l}\text { Pain significantly } \\
\text { less: } 77 \% \\
\text { Moderately less: } \\
15 \% \\
\text { Pain } \\
\text { unchanged: } 8 \%\end{array}$ & $\mathrm{~N} / \mathrm{A}$ & \\
\hline & & & & $\begin{array}{l}\text { Patient } \\
\text { satisfaction }\end{array}$ & $\mathrm{N} / \mathrm{A}$ & $\begin{array}{l}\text { Generally } \\
\text { satisfied: } 55 / 58 \\
\text { patients }\end{array}$ & $\mathrm{N} / \mathrm{A}$ & \\
\hline $\begin{array}{l}\text { Mikke } \\
\text { Isen, } \\
2015\end{array}$ & 58 & $\begin{array}{l}37.8 \\
(21- \\
54)\end{array}$ & $\mathrm{CO}_{2}$ & $\begin{array}{l}\text { Patient } \\
\text { satisfaction }\end{array}$ & $\mathrm{N} / \mathrm{A}$ & $\begin{array}{l}94.8 \%(n=55 / 58) \\
\text { of patients } \\
\text { reported a small } \\
(n=11) \text { or great } \\
\text { improvement } \\
(n=44)\end{array}$ & $\mathrm{N} / \mathrm{A}$ & $\begin{array}{l}\mathrm{CO}_{2} \text { laser surgery is an } \\
\text { effective treatment for } \\
\text { symptomatic HS lesions. } \\
\text { Patient satisfaction is high } \\
\text { despite a moderate number of } \\
\text { recurrences. }\end{array}$ \\
\hline
\end{tabular}




\begin{tabular}{|c|c|c|c|c|c|c|c|c|}
\hline & & & & $\begin{array}{l}\text { Recurrence of } \\
\text { disease }\end{array}$ & N/A & $\begin{array}{l}29.3 \%(n=17 / 58) \\
\text { of patients } \\
\text { reported } \\
\text { recurrence of } \\
\text { disease in } \\
\text { treated areas }\end{array}$ & $\mathrm{N} / \mathrm{A}$ & \\
\hline \multirow[t]{2}{*}{$\begin{array}{l}\text { Naour } \\
\text { i, } \\
2020\end{array}$} & 31 & $\begin{array}{l}\text { Not } \\
\text { specifi } \\
\text { ed }\end{array}$ & $\begin{array}{l}\text { Nd:YAG } \\
\text { laser }(4 \\
\text { treatments } \\
\text { at } 6 \text { week } \\
\text { intervals) }\end{array}$ & $\begin{array}{l}\text { Inflammatory } \\
\text { lesion count }\end{array}$ & $\begin{array}{l}\text { Week } 0 \\
\text { Untreated: } \\
5.86( \pm 6.29) \\
\text { Treated: } 5.89 \\
( \pm 4.68)\end{array}$ & $\begin{array}{l}\text { Week } 22 \\
\text { Untreated: } 1.69 \\
( \pm 15.01) \\
\text { Treated: }-1.25 \\
( \pm 10.77) \\
\text { Week } 30 \\
\text { Untreated: }-0.81 \\
\text { ( } \pm 6.08) . \\
\text { Treated: }-2.56 \\
( \pm 4.22) .\end{array}$ & $\begin{array}{l}\text { Weeks } 0-30 \\
\text { Untreated: - } \\
6.67 . \\
\text { Treated: }-8.45 .\end{array}$ & \multirow[t]{2}{*}{$\begin{array}{l}\text { The laser had no impact on } \\
\text { disease recurrence. The } \\
\text { effectiveness of laser hair } \\
\text { removal in reducing the } \\
\text { amount of inflammation } \\
\text { lesions decreases with time. }\end{array}$} \\
\hline & & & & $\begin{array}{l}\text { Proportion of } \\
\text { responders to } \\
\text { Hidradenitis } \\
\text { Suppurativa } \\
\text { Clinical } \\
\text { Response } \\
\text { (HiSCR) }\end{array}$ & $\mathrm{N} / \mathrm{A}$ & $\begin{array}{l}\text { 1-month post tx } \\
\text { Treated side: } \\
73.7 \% \\
\text { Control side: } \\
52.6 \%(\mathrm{P}= \\
0.29) . \\
\text { 3-month post tx } \\
\text { Both treated and } \\
\text { control sides: } \\
52.6 \%\end{array}$ & $\begin{array}{l}\text { No significant } \\
\text { difference in } \\
\text { number of } \\
\text { flares between } \\
\text { treated and } \\
\text { untreated sites } \\
\text { at } 1 \text { and 3- } \\
\text { month follow- } \\
\text { up }\end{array}$ & \\
\hline \multirow[t]{2}{*}{$\begin{array}{l}\text { Passe } \\
\text { ron, } \\
2009\end{array}$} & 4 & $\begin{array}{l}\text { Not } \\
\text { specifi } \\
\text { ed }\end{array}$ & $\begin{array}{l}\text { PDL-PDT } \\
\text { (ALA, only } \\
\text { one side of } \\
\text { axilla or } \\
\text { groin was } \\
\text { treated - the } \\
\text { other used } \\
\text { as control) }\end{array}$ & $\begin{array}{l}\text { Sartorius } \\
\text { score }\end{array}$ & $\begin{array}{l}\text { PDL-PDT } \\
\text { side: } 11.25 \\
\text { Control side: } \\
11\end{array}$ & $\begin{array}{l}\text { At } 1 \text { month } \\
\text { PDL-PDT side: } \\
8.25 \text {. } \\
\text { Control side: } 13 \\
\text { At } 3 \text { months } \\
\text { PDL-PDT side: } 9 \\
\text { Control side: } \\
8.67\end{array}$ & $\begin{array}{l}\text { Baseline to } \\
\text { month } 3 \\
\text { PDL-PDT } \\
\text { side: }-2.25 \\
\text { Control side: - } \\
2.33\end{array}$ & \multirow[t]{2}{*}{$\begin{array}{l}\text { PDL-PDT not effective in } \\
\text { treating HS. }\end{array}$} \\
\hline & & & & VAS & N/A & $\begin{array}{l}\text { Pain was high } \\
\text { during } \\
\text { treatment. } \\
\text { Mean: } 8(6-9)\end{array}$ & $\mathrm{N} / \mathrm{A}$ & \\
\hline $\begin{array}{l}\text { Piccol } \\
\text { o, }\end{array}$ & 2 & $\begin{array}{l}32 \\
(26-\end{array}$ & $\begin{array}{l}\text { IPL (6 } \\
\text { sessions) }\end{array}$ & $\begin{array}{l}\text { Sartorius } \\
\text { score }\end{array}$ & $30(24-36)$ & $\mathrm{N} / \mathrm{A}$ & $\mathrm{N} / \mathrm{A}$ & \multirow{2}{*}{$\begin{array}{l}\text { HS lesions were completely } \\
\text { resolved, along with achieving } \\
\text { hair removal. }\end{array}$} \\
\hline 2014 & & 38) & & Recurrence & $\mathrm{N} / \mathrm{A}$ & $\begin{array}{l}\text { HS pustular } \\
\text { papules } \\
\text { resolved post } \\
\text { treatment }\end{array}$ & $N / A$ & \\
\hline $\begin{array}{l}\text { Rodri } \\
\text { guez- } \\
\text { Prieto } \\
\text {, } 2013\end{array}$ & 3 & $\begin{array}{l}47 \\
(36- \\
62)\end{array}$ & $\begin{array}{l}\text { PDT } \\
\text { (intralesional } \\
5-A L A \text { and } \\
\text { irradiated } \\
\text { with diode } \\
\text { laser) }\end{array}$ & $\begin{array}{l}\text { Recurrence of } \\
\text { disease }\end{array}$ & $\mathrm{N} / \mathrm{A}$ & $\begin{array}{l}\text { Patient 1: No } \\
\text { recurrence at } 9 \\
\text { months } \\
\text { Patient 2: No } \\
\text { recurrence after } \\
14 \text { months } \\
\text { Patient 3: } \\
\text { resolution of } \\
\text { symptoms after } \\
7 \text { months }\end{array}$ & $\mathrm{N} / \mathrm{A}$ & $\begin{array}{l}\text { No recurrence post } \\
\text { intralesional PDT in all } 3 \\
\text { patients. }\end{array}$ \\
\hline $\begin{array}{l}\text { Share } \\
\text { ef, } \\
2011\end{array}$ & 13 & $\begin{array}{l}35 \\
(25- \\
66)\end{array}$ & $\begin{array}{l}\text { PUVA } \\
\text { (bath) }\end{array}$ & $\begin{array}{l}\text { DLQI } \\
\text { Disease } \\
\text { clearance }\end{array}$ & $\begin{array}{l}16(11-26) \\
\mathrm{N} / \mathrm{A}\end{array}$ & $\begin{array}{l}\text { N/A } \\
\text { Clear/near clear: } \\
5 \text { patients; } \\
\text { moderate } \\
\text { clearance: } 4 ; \text { no- } \\
\text { minimal: } 4\end{array}$ & $\begin{array}{l}\mathrm{N} / \mathrm{A} \\
\mathrm{N} / \mathrm{A}\end{array}$ & $\begin{array}{l}\text { Bath PUVA is possibly an } \\
\text { effective treatment modality for } \\
\text { some people with HS. }\end{array}$ \\
\hline \multirow{4}{*}{$\begin{array}{l}\text { Sotiri } \\
\text { ou, } \\
2009\end{array}$} & 5 & $\begin{array}{l}33.6 \\
(25-\end{array}$ & $\begin{array}{l}\text { PDT } \\
\text { (topical 5- }\end{array}$ & $\begin{array}{l}\text { Sartorius } \\
\text { score }\end{array}$ & 18.8 & 17.2 & -1.6 & \multirow[t]{4}{*}{$\begin{array}{l}\text { No significant improvement } \\
\text { post treatment. }\end{array}$} \\
\hline & & 43) & ALA) & $\begin{array}{l}\text { VAS - disease } \\
\text { severity and } \\
\text { pain }\end{array}$ & 2.4 & 2.1 & -0.3 & \\
\hline & & & & DLQI & N/A & $\mathrm{N} / \mathrm{A}$ & $\begin{array}{l}\text { Reduction in } \\
\text { mean of } 6.4 \%\end{array}$ & \\
\hline & 4 & & $\begin{array}{l}\text { PDT } \\
\text { (topical ALA) }\end{array}$ & $\begin{array}{l}\text { Sartorius } \\
\text { score }\end{array}$ & 17.8 & 23.3 & 5.5 & \\
\hline
\end{tabular}




\begin{tabular}{|c|c|c|c|c|c|c|c|c|}
\hline $\begin{array}{l}\text { Strau } \\
\text { ss, }\end{array}$ & & $\begin{array}{l}\text { Not } \\
\text { specifi }\end{array}$ & & $\begin{array}{l}\text { VAS - disease } \\
\text { severity }\end{array}$ & 8.1 & 7.1 & -1 & \multirow{3}{*}{$\begin{array}{l}\text { No significant improvement } \\
\text { post treatment. Worsening } \\
\text { symptoms for } 2 \text { patients. } \\
\text { Resolution of disease in } 3 / 3 \\
\text { patients. No recurrence noted } \\
\text { at follow up. }\end{array}$} \\
\hline 2005 & & & & VAS - pain & 7 & 5.3 & -1.7 & \\
\hline $\begin{array}{l}\text { Suare } \\
\text { z- } \\
\text { Vallad } \\
\text { ares, } \\
2018\end{array}$ & 5 & $\begin{array}{l}\text { Not } \\
\text { specifi } \\
\text { ed }\end{array}$ & $\begin{array}{l}\text { PDT } \\
\text { (intralesional } \\
\text { ALA) }\end{array}$ & $\begin{array}{l}\text { Qualitative } \\
\text { descriptions } \\
\text { by } \\
\text { investigators }\end{array}$ & $\mathrm{N} / \mathrm{A}$ & $\begin{array}{l}\text { All patients } \\
\text { achieved a } \\
\text { complete } \\
\text { response in the } \\
\text { treated lesion }\end{array}$ & $\mathrm{N} / \mathrm{A}$ & \\
\hline \multirow[t]{3}{*}{$\begin{array}{l}\text { Suare } \\
\text { z- } \\
\text { Vallad } \\
\text { ares, } \\
2017\end{array}$} & \multirow[t]{3}{*}{38} & \multirow[t]{3}{*}{$\begin{array}{l}36 \\
(30- \\
44)\end{array}$} & \multirow[t]{3}{*}{$\begin{array}{l}\text { I-PDT } \\
(5-A L A)\end{array}$} & $\begin{array}{l}\text { Response to } \\
\text { treatment }\end{array}$ & $\mathrm{N} / \mathrm{A}$ & $\begin{array}{l}\text { Complete } \\
\text { response: } 29 \\
\text { patients } \\
\text { Persistence: } 8 \\
\text { patients } \\
\text { Recurrence: } 1 \\
\text { patient }\end{array}$ & $\mathrm{N} / \mathrm{A}$ & \multirow[t]{3}{*}{$\begin{array}{l}\text { A complete response (no } \\
\text { lesions or symptoms) rate of } \\
76.3 \% \text {. I-PDT is a potential } \\
\text { effective alternative treatment } \\
\text { to HS. }\end{array}$} \\
\hline & & & & DLQI & $\begin{array}{l}\text { Median = } 10 \\
(7-17)\end{array}$ & $\begin{array}{l}\text { Median = } 1(0- \\
2.25)\end{array}$ & -9 & \\
\hline & & & & HSS & $\begin{array}{l}\text { Median = } 28.5 \\
(11.75-38.5)\end{array}$ & $\begin{array}{l}\text { Median = } 0(0- \\
45)\end{array}$ & -28.5 & \\
\hline \multirow[t]{2}{*}{$\begin{array}{l}\text { Theut } \\
\text { Riis, } \\
2018\end{array}$} & \multirow[t]{2}{*}{25} & \multirow[t]{2}{*}{$\begin{array}{l}39.2 \\
(\mathrm{SD} \\
10.9 / 1 \\
7-63)\end{array}$} & \multirow[t]{2}{*}{ IPL } & $\begin{array}{l}\text { Patient } \\
\text { reported } \\
\text { disease } \\
\text { activity }\end{array}$ & $\mathrm{N} / \mathrm{A}$ & $\begin{array}{l}\text { Reduced } \\
\text { disease activity: } \\
\text { 13/25 patients }\end{array}$ & $\mathrm{N} / \mathrm{A}$ & \multirow{2}{*}{$\begin{array}{l}\text { IPL can be used as an } \\
\text { adjuvant treatment for HS - in } \\
\text { those with mild to moderate } \\
\text { disease with minimal scar } \\
\text { tissue. }\end{array}$} \\
\hline & & & & Hair reduction & $\mathrm{N} / \mathrm{A}$ & $\begin{array}{l}\text { Effect on hair in } \\
17 / 25 \text { patients }\end{array}$ & $\mathrm{N} / \mathrm{A}$ & \\
\hline \multirow{3}{*}{$\begin{array}{l}\text { Vallad } \\
\text { ares- } \\
\text { Narga } \\
\text { nes, } \\
2015\end{array}$} & \multirow[t]{3}{*}{27} & \multirow[t]{3}{*}{$\begin{array}{l}30.3 \\
(19- \\
62)\end{array}$} & \multirow[t]{3}{*}{$\begin{array}{l}\text { I-PDT (5- } \\
\text { ALA) }\end{array}$} & $\begin{array}{l}\text { Modified } \\
\text { Sartorius } \\
\text { score }\end{array}$ & 20.67 & 8.81 & -11.86 & \multirow{3}{*}{$\begin{array}{l}21 / 27 \text { patients had either a } \\
\text { good or complete response to } \\
\text { treatment. Intralesional } \\
\text { application of PDT allows the } \\
\text { light to reach various depths } \\
\text { with the lowest amount of } \\
\text { energy needed. It may be an } \\
\text { effective treatment for HS. }\end{array}$} \\
\hline & & & & $\begin{array}{l}\text { Response to } \\
\text { treatment }\end{array}$ & $\mathrm{N} / \mathrm{A}$ & $\begin{array}{l}\text { Complete } \\
\text { response: } 10 \\
(37 \%) \\
\text { Good response: } \\
11(41 \%) \\
\text { Partial response: } \\
5(19 \%)\end{array}$ & $\mathrm{N} / \mathrm{A}$ & \\
\hline & & & & VAS - pain & $N / A$ & $\begin{array}{l}\text { Severe pain }(9) \text { : } \\
1 \\
\text { Moderate }(6-9) \text { : } \\
4 \\
\text { Low }(<6): 22\end{array}$ & $\mathrm{~N} / \mathrm{A}$ & \\
\hline \multirow[t]{2}{*}{$\begin{array}{l}\text { Vilarr } \\
\text { asa, } \\
2019\end{array}$} & \multirow[t]{2}{*}{28} & \multirow[t]{2}{*}{46} & \multirow[t]{2}{*}{$\begin{array}{l}\text { PDT (topical } \\
\text { 5-ALA) }\end{array}$} & $\begin{array}{l}\text { DLQI \& EVA } \\
\text { (pain visual } \\
\text { scale) }\end{array}$ & $\mathrm{N} / \mathrm{A}$ & $\mathrm{N} / \mathrm{A}$ & $\begin{array}{l}\text { All patients } \\
\text { showed } \\
\text { improvement } \\
\text { in DLQI \& EVA }\end{array}$ & \multirow{2}{*}{$\begin{array}{l}\text { All patients improved in DLQI } \\
\text { and EVA scores } 8 \text { weeks post } \\
\text { last treatment session. } \\
\text { Ultrasound showed a } \\
\text { resolution in the lesions of } 13 \\
\text { patients, partial resolution in } \\
12 \text { patients, and poor in } 3 .\end{array}$} \\
\hline & & & & Ultrasound & $\mathrm{N} / \mathrm{A}$ & $\begin{array}{l}\text { Resolution of } \\
\text { lesions: } 13 \\
\text { patients } \\
\text { Partial } \\
\text { resolution: } 12 \\
\text { patients } \\
\text { Poor: } 3 \text { patients }\end{array}$ & $N / A$ & \\
\hline \multirow[t]{3}{*}{$\begin{array}{l}\text { Vosse } \\
\text { n, } \\
2018\end{array}$} & \multirow[t]{3}{*}{15} & \multirow[t]{3}{*}{$\begin{array}{l}34.1 \\
(10.1)\end{array}$} & \multirow[t]{3}{*}{$\begin{array}{l}\text { Nd:YAG } \\
\text { laser (hair } \\
\text { removal on } \\
\text { patients with } \\
\text { mild HS) }\end{array}$} & $\begin{array}{l}\text { Number of HS } \\
\text { flares/month } \\
\text { (Questionnaire } \\
\text { ) }\end{array}$ & $\begin{array}{l}<1 \text { flare: } 4 \\
\text { patients } \\
1 \text { flare: } 2 \\
\text { patients } \\
2 \text { flares: } 1 \\
\text { patient } \\
3 \text { flares: } 3 \\
\text { patients } \\
>3 \\
\text { flares/continuo } \\
\text { us } \\
\text { inflammation: } \\
5 \text { patients }\end{array}$ & $\begin{array}{l}<1 \text { flare: } 8 \\
\text { patients } \\
1 \text { flare: } 0 \\
2 \text { flares: } 3 \\
\text { patients } \\
3 \text { flares: } 3 \\
\text { patients } \\
\text { > } 3 \text { flares/ } \\
\text { continuous } \\
\text { inflammation: } 1 \\
\text { patient }\end{array}$ & $\begin{array}{l}\text { Decrease in } \\
\text { number of } \\
\text { monthly flares } \\
(p=0.019) .\end{array}$ & \multirow[t]{3}{*}{$\begin{array}{l}\text { Laser hair removal can be } \\
\text { used to prevent the } \\
\text { progression of disease. } \\
\text { Results include decrease in } \\
\text { the number of monthly flares, } \\
\text { average HS disease severity } \\
\text { was significantly lower post } \\
\text { treatment (NRS } 6.4 \pm 2.8 \\
\text { versus NRS } 3.6 \pm 3.5) \text {. Overall } \\
\text { treatment satisfaction was } \\
\text { rated with a NRS score of } 6.7 \\
\pm 2.4 .2 / 3 \text { patients would } \\
\text { recommend the treatment. }\end{array}$} \\
\hline & & & & $\begin{array}{l}\text { NRS disease } \\
\text { severity }(0-10)\end{array}$ & $\begin{array}{l}\text { NRS } 6.4 \\
( \pm 2.8)\end{array}$ & $\begin{array}{l}\text { NRS } 3.6( \pm 3.5) \\
p=0.01\end{array}$ & -2.8 & \\
\hline & & & & $\begin{array}{l}\text { NRS overall } \\
\text { treatment } \\
\text { satisfaction (0- } \\
10)\end{array}$ & $\mathrm{N} / \mathrm{A}$ & NRS $6.7( \pm 2.4)$ & $\mathrm{N} / \mathrm{A}$ & \\
\hline
\end{tabular}

November $2021 \quad$ Volume 5 Issue 6 


\begin{tabular}{|c|c|c|c|c|c|c|c|c|}
\hline \multirow[t]{2}{*}{$\begin{array}{l}\text { Wilde } \\
\text { n, } \\
2019\end{array}$} & 13 & $\begin{array}{l}38 \\
923- \\
57)\end{array}$ & $\begin{array}{l}\text { IPL and } \\
\text { radiofrequen } \\
\text { cy }(R F)\end{array}$ & $\begin{array}{l}\text { Active lesions } \\
\text { count }\end{array}$ & $\mathrm{N} / \mathrm{A}$ & $\mathrm{N} / \mathrm{A}$ & $\begin{array}{l}\text { IPL: } 0.8 \\
\text { RF group: }-0.4 \\
\text { IPL+RF group: } \\
-1.3 \\
\text { Whole cohort } \\
\text { on crossover } \\
\text { to IPL+RF for } \\
24 \text { weeks: }-3.6 \\
(p=0.001) \text {. }\end{array}$ & $\begin{array}{l}\mathrm{IPL}+\mathrm{RF} \text { is a promising } \\
\text { treatment for HS, increasing in } \\
\text { effect over time and without } \\
\text { severe side effects. }\end{array}$ \\
\hline & & & & DLQI & $\mathrm{N} / \mathrm{A}$ & $\mathrm{N} / \mathrm{A}$ & $\begin{array}{l}\text { IPL group: }-1.3 \\
\text { RF group: }-5.1 \\
\text { IPL+ RF } \\
\text { group: }-6.6 \\
\text { ( } p=0.025) \text {. } \\
\text { Whole cohort } \\
\text { on crossover } \\
\text { to IPL+RF for } \\
24 \text { weeks: }-5.2 \\
\text { ( } p=0.003) \text {. }\end{array}$ & \\
\hline $\begin{array}{l}\text { Wollin } \\
\text { a, } \\
2004\end{array}$ & 17 & $\begin{array}{l}(29- \\
41)\end{array}$ & $\begin{array}{l}\text { Transdermal } \\
\mathrm{CO}_{2}\end{array}$ & $\begin{array}{l}\text { Clinical } \\
\text { response }\end{array}$ & $\mathrm{N} / \mathrm{A}$ & $\mathrm{N} / \mathrm{A}$ & $\begin{array}{l}\text { Improvement } \\
\text { of granulation } \\
\text { and reduction } \\
\text { of discharge } \\
\text { and malodor } \\
1 \text {-week post } \\
\text { treatment }\end{array}$ & \\
\hline $\begin{array}{l}\text { Xu, } \\
2011\end{array}$ & 19 & $\begin{array}{l}37 \\
(23- \\
54)\end{array}$ & $\begin{array}{l}\text { 1064nm } \\
\text { Nd:YAG } \\
\text { laser }\end{array}$ & $\begin{array}{l}\text { Lesion Area } \\
\text { and Severity } \\
\text { Index (LASI) }\end{array}$ & $\mathrm{N} / \mathrm{A}$ & $\mathrm{N} / \mathrm{A}$ & $\begin{array}{l}\text { All sites: } \\
-31.6 \% \\
(p<0.05) \\
\text { Axillary sites: } \\
-24.4 \% \\
\text { ( } p=0.08) \\
\text { Inguinal site: } \\
-36.8 \% \\
(p=0.001)\end{array}$ & $\begin{array}{l}\text { The percentage change in } \\
\text { modified HS-LASI score after } \\
2 \text { months was }-31.6 \% \\
\text { ( } p<.001 \text { ) averaged over all } \\
\text { anatomic sites. HS-LASI } \\
\text { scores trended down from } \\
\text { baseline to } 1 \text { month and } 2 \\
\text { months after treatment for both } \\
\text { axilla and inguinal sites. }\end{array}$ \\
\hline $\begin{array}{l}\text { Zhang } \\
, 2016\end{array}$ & 3 & $\begin{array}{l}(17- \\
38)\end{array}$ & PDT (ALA) & DLQI & $26.67(1.15)$ & $\begin{array}{l}\text { Session 1: } 24.67 \\
\text { Session 2: } 24.33 \\
\text { Session 3: } 24.33\end{array}$ & $\mathrm{~N} / \mathrm{A}$ & $\begin{array}{l}\text { No improvement for late-stage } \\
\text { HS. }\end{array}$ \\
\hline
\end{tabular}

DLQI: Dermatology Life Quality Index; HSS: Hidradenitis severity score; MAL: methyl amino; N: number of participants; PDT: photodynamic therapy I-PDT: intralesional photodynamic therapy PUVA: psoralen plus ultraviolet A; US: ultrasonographic; VAS: visual analogue scale; VSS: Vancouver Scar Scale; QOL: quality of life

MCID: minimum clinically important difference NRS $30-30 \%$ reduction to baseline numerical rating scorel

\# tetracycline used as a photosensitizer combined with $600 \mathrm{~nm}$ red light 